\title{
Development and validation of a nomogram combining hematological and imaging features for preoperative prediction of microvascular invasion in hepatocellular carcinoma patients
}

\author{
Qiang Zhou ${ }^{1 \#}$, Chenhao Zhou ${ }^{1,2 \#}$, Yirui Yin ${ }^{1,3 \#, ~ W a n y o n g ~ C h e n ~}{ }^{4}$, Chunxiao Liu ${ }^{2}$, Manar Atyah ${ }^{1}$, Jialei \\ Weng $^{1}$, Yinghao Shen ${ }^{1}$, Yong $\mathbf{Y i}^{1}$, Ning Ren ${ }^{1,4}$ \\ ${ }^{1}$ Department of Liver Surgery, Liver Cancer Institute, Zhongshan Hospital, and Key Laboratory of Carcinogenesis and Cancer Invasion (Ministry of \\ Education), Fudan University, Shanghai, China; ${ }^{2}$ Department of Molecular and Cellular Oncology, The University of Texas MD Anderson Cancer \\ Center, Houston, Texas, USA; ${ }^{3}$ Department of Liver Surgery, Xiamen Branch, Zhongshan Hospital, Fudan University, Xiamen, China; ${ }^{4}$ Institute of \\ Fudan Minhang Academic Health System, and Key Laboratory of Whole-period Monitoring and Precise Intervention of Digestive Cancer (SMHC), \\ Minhang Hospital \& AHS, Fudan University, Shanghai, China \\ Contributions: (I) Conception and design: N Ren, Y Yi, W Chen, C Zhou, Y Yin, Q Zhou; (II) Administrative support: N Ren, Y Yi, Y Shen; (III) \\ Provision of study materials or patients: N Ren, Y Yi; (IV) Collection and assembly of data: M Atyah, Y Shen, J Weng; (V) Data analysis and \\ interpretation: C Liu, Y Yin, Q Zhou; (VI) Manuscript writing: All authors; (VII) Final approval of manuscript: All authors. \\ \#These authors contributed equally to this work. \\ Correspondence to: Ning Ren. Department of Liver Surgery, Liver Cancer Institute, Zhongshan Hospital, Fudan University, 180 Fenglin Road, \\ Shanghai 200032, China. Email: ren.ning@zs-hospital.sh.cn; Yong Yi. Department of Liver Surgery, Liver Cancer Institute, Zhongshan Hospital, \\ Fudan University, 180 Fenglin Road, Shanghai 200032, China. Email: yi.yong@zs-hospital.sh.cn.
}

Background: Microvascular invasion (MVI) is a significant hazard factor that influences the recurrence and survival of hepatocellular carcinoma (HCC) patients after undergoing hepatectomy. This study aimed to develop and validate a nomogram that combines hematological and imaging features of HCC patients to preoperatively predict MVI, and investigate the effect of wide resection margin $(\geq 1 \mathrm{~cm})$ on the prognosis of MVI-positive HCC patients.

Methods: A total of 709 HCC patients who underwent hepatectomy at the Liver Cancer Institute of Zhongshan Hospital, Fudan University between June 1, 2015 and December 30, 2016 were included in this study and divided into training (496 patients) and validation cohort (213 patients). Least absolute shrinkage and selection operator (Lasso) regression and multivariable logistic regression were used for variables' selection and development of the predictive model. The model was presented as a nomogram, and its performance was assessed in terms of discrimination, calibration and clinical usefulness.

Results: Independent prognostic factors such as alkaline phosphatase (ALP, >125 U/L), alpha-fetoprotein (AFP, within 20-400 or >400 ng/mL), protein induced by vitamin $\mathrm{K}$ absence-II (PVIKA-II, within 40-400 or $>400 \mathrm{mAU} / \mathrm{mL}$ ), tumor number, diameter, pseudo-capsule, tumor growth pattern and intratumor hemorrhage were incorporated in the nomogram. The model showed good discrimination and calibration, with a concordance index $(0.82,95 \% \mathrm{CI}, 0.782-0.857)$ in the training cohort and C-index $(0.80,95 \% \mathrm{CI}$, $0.772-0.837$ ) in the validation cohort. Decision curve analysis (DCA) also showed that this model is clinically useful. Moreover, HCC patients with wide resection margin had a significantly lower 3-year recurrence rate than those with narrower resection margin $(0.5-1 \mathrm{~cm})$.

Conclusions: This study presents an optimal model for preoperative prediction of MVI and shows that wide resection margin for MVI-positive HCC patients has a better prognosis. This model can help surgeons choose the best treatment options for HCC patients before and after the operation.

Keywords: Hepatocellular carcinoma (HCC); microvascular invasion (MVI); nomogram; prediction; disease-free survival (DFS) 
Submitted Jun 14, 2020. Accepted for publication Nov 22, 2020.

doi: 10.21037/atm-20-4695

View this article at: http://dx.doi.org/10.21037/atm-20-4695

\section{Introduction}

Hepatocellular carcinoma (HCC) is one of the most common malignant tumors in the world. In early 2020, the latest statistics report on cancer in the United States showed that HCC was the fifth leading cause of tumor death with a 5 -year survival rate of only $18 \%$ (1). Recently, early clear diagnosis and combinations of comprehensive treatment (such as surgery and molecular targeted therapies) have improved the prognosis of HCC patients. However, the recurrence rate at 2 years after liver cancer resection is still close to $50 \%$, and as high as $75 \%$ at 5 years (2).

MVI is a well-known risk factor that affects the recurrence of HCC and survival of patients after hepatectomy, and is included in the latest TNM staging system (3). However, MVI cannot be distinguished preoperatively as it is only diagnosed by post-operative pathological examination. Hence, it is essential to establish an efficient predictive model that distinguishes MVI preoperatively by combining hematological and imaging data.

A variety of previous studies concentrated on highly reliable factors to predict MVI. Xu et al. built a radiographic-radiomic (RR) model with a high capacity to preoperatively predict MVI from thousands of radiomic features (based on contrast-enhanced CT) (4). Lee et al. found hypo-intensity in hepatobiliary phase (HBP) can be utilized as a specific imaging biomarker for estimating MVI through gadoxetic acid-enhanced MRI (5). However, contrast-enhanced CT is not as sensitive as MRI in detecting early HCC. MRI has become the mainstream technology in the diagnosis of HCC. Gadoxetic acidenhanced MRI has not been extensively used for HCC detection because of its expensive contrast agent. Wang et al. also applied diffusion kurtosis imaging (DKI)-derived parameters of MVI for preoperative assessment, but the sample size was too small (6). Banerjee et al. combined imaging features and gene expression to build a radiogenomic venous-invasion (RVI) predictor for MVI. However, these criteria have not been well-established (7). Recently, PIVKA-II was adopted as a biomarker for the early detection of HCC (8). Other studies also showed that it can be used as a predictive biomarker of MVI (9). However, more clinical studies are needed to verify it, and clarify its effective cut-off.
Complete surgical resection is the most effective therapy for curing HCC (10). Advanced surgical techniques, such as anatomical resection and widening resection margin can theoretically improve the radical of HCC, but there is no unified understanding regarding this issue $(11,12)$. Accurate evaluation of the efficacy of HCC surgical methods is still a question worthy of further clinical investigation. MVI may be one factor affecting the thoroughness of radical operation. Therefore, this study investigated the effect of resection with wide margin on the prognosis of MVIpositive HCC patients. The findings of this study can provide theoretical basis for different surgical methods and assist surgeons with choosing whether a wide resection margin should be adopted. For advanced HCC stages, it can also assist surgeons with choosing an appropriate treatment strategy such as neoadjuvant therapy or molecular targeted therapy.

Therefore, the aim of this study was to build a specific and practical model for accurate preoperative prediction of MVI. This model combined hematological tests (including inflammatory and tumor markers of HCC) and imaging features of contrast-enhanced MRI, which are accessible to surgeons. It can guide surgeons to provide successful treatment and clinical management for HCC patients and reduce the risk of recurrence. Therefore, this model we have established is more suitable and practical for surgeons.

The presentation of the following article is in accordance with the transparent reporting of a multivariable prediction model for individual prognosis or diagnosis (TRIPOD) reporting checklist (available at http://dx.doi.org/10.21037/ atm-20-4695).

\section{Methods}

\section{Patients enrollment}

A total of 709 HCC patients (aged 18 years or older) were enrolled in the study. All patients underwent resection at the "Liver Cancer Institute of Zhongshan Hospital, Fudan University" between June 1, 2015 and December 30, 2016 (496 as training validation cohort and 213 as validation cohort). All curative-intent hepatectomy procedures were performed by experienced hepatobiliary surgeons, and surgical specimens were histopathologically confirmed. The 
study was conducted in accordance with the "Declaration of Helsinki" (as revised in 2013). Clinical data in the study was obtained from Zhongshan hospital medical system. The study was approved by the ethics committee of Zhongshan Hospital, Fudan University. Approval No. is Y2018-155. Informed consent of clinical data used in this study were obtained from all patients without financial compensation.

The exclusion criteria were as followed: (I) patients younger than 18 years old; (II) patients with Nodules $>10 \mathrm{~cm}$, Child-Pugh C, preoperative anticancer treatment, metastasis, macroscopic portal vein tumor thrombus (MAPVTT), other cancers history, incomplete clinical data, or incomplete radical resection; (III) patients who died within 30 days after surgery.

\section{Preoperative management and surgical procedure}

Patients' general condition and liver reserve function were comprehensively evaluated preoperatively. The functional status score proposed by the "Eastern Cooperative Oncology Group" (ECOG) was often used to evaluate the patient's general condition. Liver functions were evaluated by Child-Pugh score and indocyanine green (ICG) clearance test or transient elastography to measure liver stiffness $(13,14)$. Other routine preoperative laboratory examinations included renal, cardiopulmonary $(>60$ years old) function tests, routine blood tests, hepatitis B and C immunology, tumor Markers, and coagulation function. The criteria of the "American Association for the Study of Liver Diseases" (AASLD) Development and validation of a nomogram combining hematological and imaging features for preoperative prediction of microvascular invasion (MVI) in HCC patients used for the diagnosis of HCC. Anatomical and non-anatomical resections are common surgical techniques. The most suitable resection margin was implemented according to the specific location of the tumor. According to the different surgical resection margins, patients were divided into wide resection margin group $(\geq 1 \mathrm{~cm})$ and narrow resection margin group $(0.5-1 \mathrm{~cm})$. Patients received good management after hepatectomy until their discharge by a standard protocol.

\section{Clinicopathologic variables}

Demographic and clinicopathological data are shown in Table 1. The imaging data and morphologic features included maximum tumor length, tumor margin ( 0 for smooth margin and 1 for non-smooth margin), tumor growth pattern ( 0 for regular or round morphology and 1 for Infiltrative border with irregular shape), cirrhosis (0 for absence and 1 for presence), pseudo-capsules (defined as the enhancement in arterial phase, portal phase or delay phase, represented with 0 for well-defined pseudo-capsules and 1 for ill-defined pseudo-capsules), intratumor inhomogeneous imaging or with necrosis and hemorrhage ( 0 for absence and 1 for presence), arterial enhancement ( 0 for hyperenhancement and 1 for hypo-/mild enhancement) (15), number of tumors detected ( 0 for single and 1 for multiple), and hypo-intensity in portal phase or delay phase (Washout) (0 for absence and 1 for presence) $(16,17)$.

All cases were evaluated preoperatively by two experienced radiologists and any disagreement between them were discussed until a final standard radiologic report was generated.

\section{Histopathology}

Surgical specimens were evaluated by a dedicated and experienced pathologist. Pathologic characteristics included: (I) histologic tumor grade, classified according to Edmondson-Steiner grade four-classification method (I-IV); (II) histological morphology, such as thin beam, thick beam, pseudo-glandular tube and patch type; (III) special types of HCC, including steatosis, clear cell, mega-beam mass, sclerosis, photic cell, fibrous layer, neutrophil, and lymphocyte type; (IV) tumor growth patterns, such as pericarcinoma invasion, breakthrough of capsules, MVI, and satellite nodules.

MVI was defined as the microscopic presence of tumor cell nests in the vascular cavity lined by endothelial cells (3). Pathological grading method was M0 when no MVI was found, M1 (low-risk group) when less than 5 MVI were found and occurred in peritumor; M2 (high-risk group) when more than 5 MVI were found or MVI occurred in distant liver tissues.

\section{Follow-up}

After undergoing hepatectomy, all patients were monitored for 1 month and then every 3 months using color doppler ultrasonography or MRI and tumor markers. New tumor presented in imaging studies was defined as HCC recurrence. Patients were followed-up until December 30, 2019. 
Table 1 Clinical characteristics of patients in the training and validation cohort

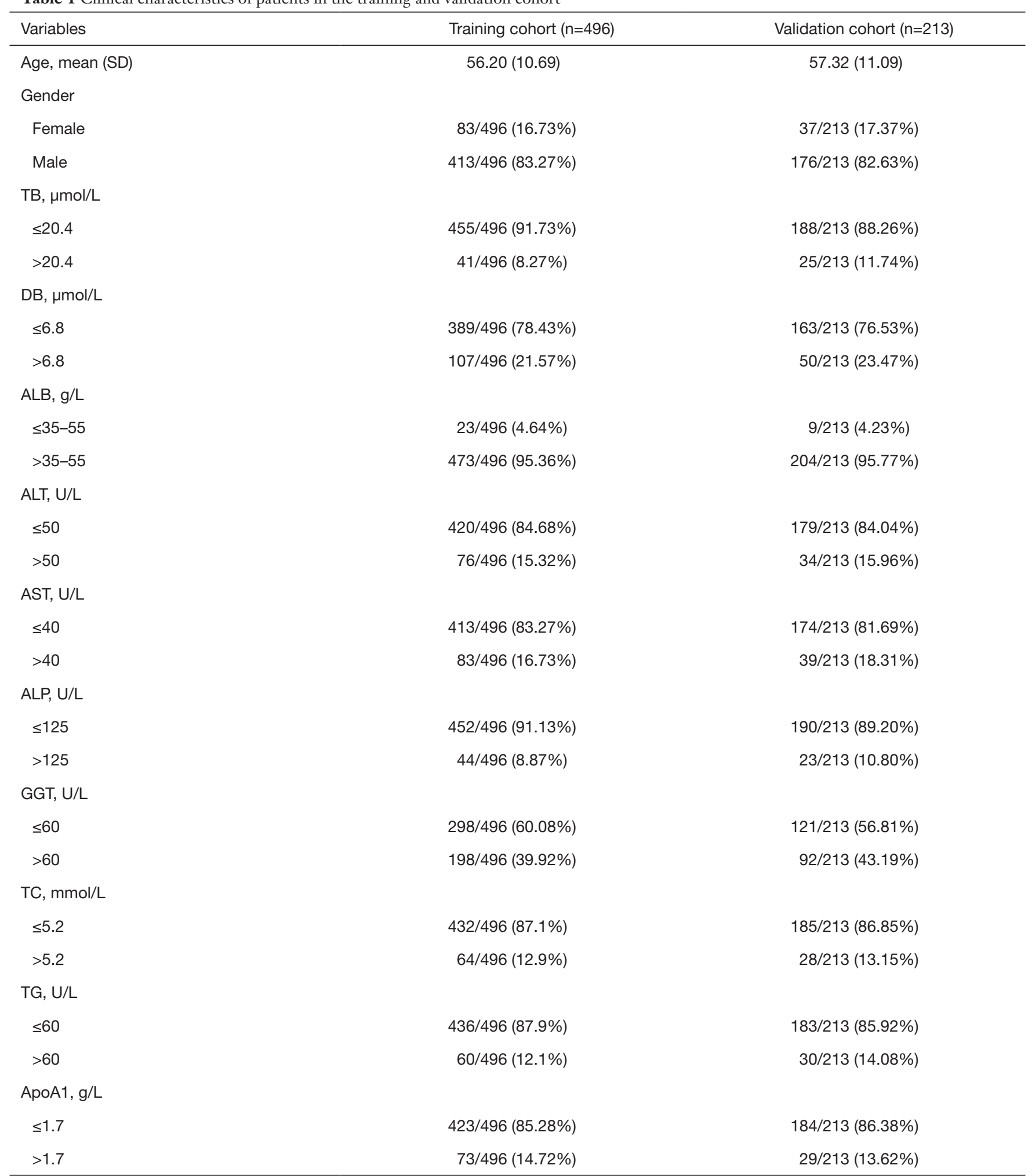

Table 1 (continued) 
Table 1 (continued)

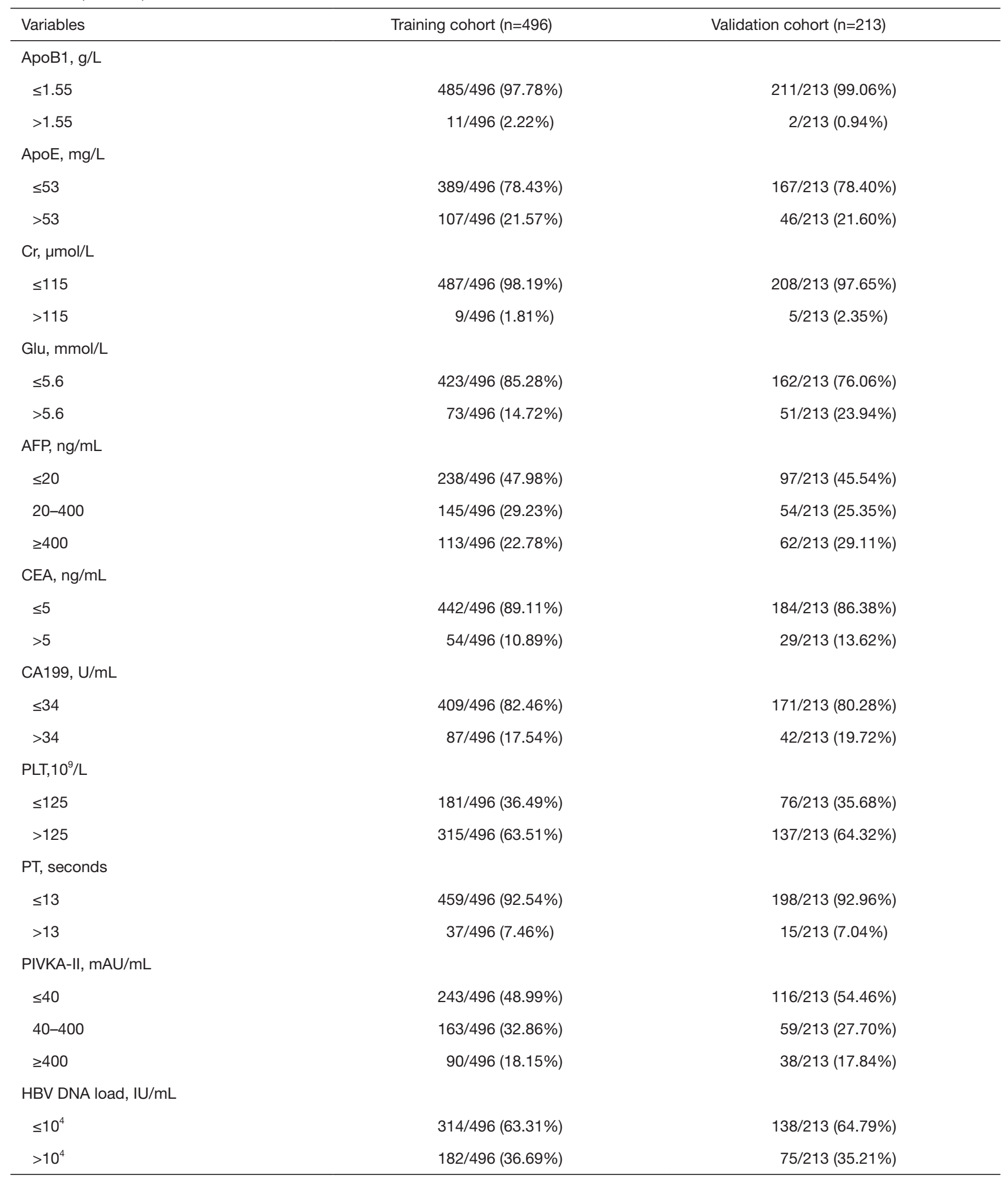

Table 1 (continued) 
Table 1 (continued)

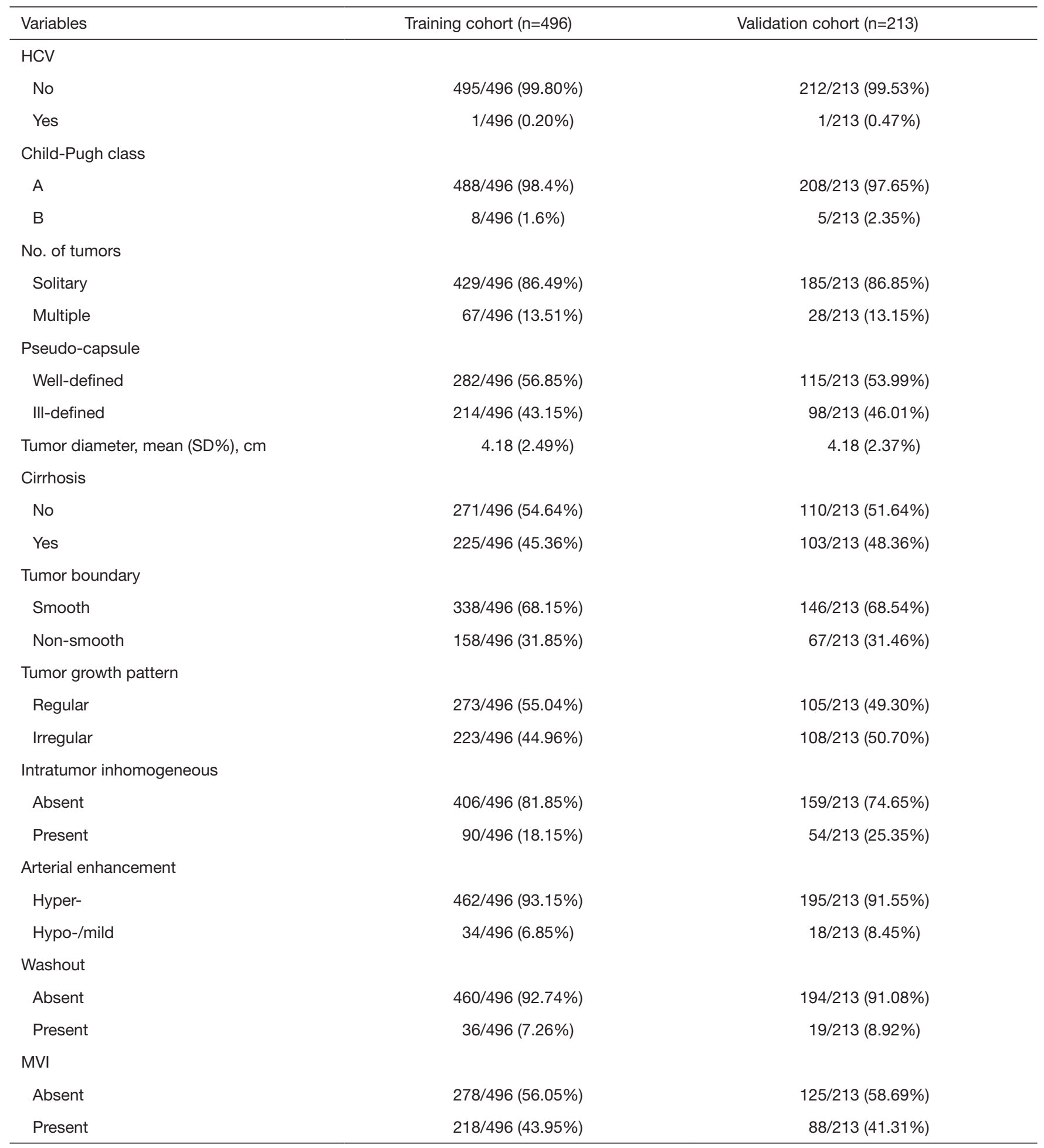

TB, total bilirubin; DB, direct bilirubin; ALB, albumin; ALT, alanine transaminase; AST, aspartate transaminase; ALP, alkaline phosphatase; GGT, glutamyl transpeptidase; TC, total cholesterol; TG, triglyceride; APoA1, apolipoprotein A; APoB1, apolipoprotein B; ApoE, apolipoprotein e; Cr, Creatinine; Glu, fasting plasma glucose; AFP, alpha-fetoprotein; CEA, carcino-embryonic antigen; CA199, carbohydrate antigen; PLT, blood platelet; PT, prothrombin time; PIVKA-II, protein induced by vitamin K absence-II; HBV, hepatitis B virus; $\mathrm{HCV}$, hepatitis $\mathrm{C}$ virus. 


\section{Statistical analysis}

Categorical variables were reported as whole numbers and/ or proportions, and continuous variables were reported as medians with standard deviation. Interobserver agreement for the presence of MRI imaging features was evaluated as Cohen $\kappa$ coefficient. Clinicopathological variables associated with MVI were selected and assessed based on scientific knowledge, clinical discovery and markers' identification in previous studies. Categorical variables between HCC patients with or without MVI were compared using Chi square test or Fisher exact test and continuous variables by using two-sample $t$-test or Mann-Whitney $\mathrm{U}$ test. The collinearity of variables or plausible interaction terms including age, AFP, PIVKA-II, tumor diameter, capsules, tumor growth pattern, intratumor necrosis or hemorrhage were evaluated. No interaction term was found in these variables. Continuous predictors (e.g., AFP and PIVKA-II) were categorized based on previous research $(5,18)$. LASSO and 10 -fold cross-validation were suitable for the regression of 34 clinicopathological variables associated with MVI and used to select the most effective and essential predictive features from the training cohort (Appendix 1). Selected variables were integrated in multivariate regression logistic analysis. Hazard ratios (HRs) were presented with their 95\% CIs. Nomogram was used to predict the probability of MVI (19). Regression coefficients were converted proportionally to a 0 to 100 -point scale. Total points were obtained by adding independent variables derived score to define the linear predictor (20).

The discrimination ability (predictive accuracy for individual outcomes) of the model was evaluated using the area under receiver operating characteristic curve (AUC) or C index (21). The C index is equivalent to the AUC. The accuracy of the model estimating outcomes function (calibration) was evaluated using calibration plot. The performance of the nomogram was tested in the validation cohort by calibration curve and AUC. Clinical usefulness of the nomogram was estimated using decision curve analysis (DCA) by quantifying the net benefits compared to other predictive models (based on only hematological or imaging features) (Appendix 2) (22). The index integrated discrimination improvement (IDI) and the net reclassification improvement (NRI) were used to evaluate the improvement in the predictive accuracy. Figures were drawn with R-Studio for windows (Version 3.6.2, http:// www.r-project.org). The packages of $\mathrm{R}$ used were show in Table S1. All tests were two sided, and $\mathrm{P}<0.05$ was considered statistically significant.

\section{Results}

\section{Clinicopathologic characteristics}

A total of 709 HCC patients who met the inclusion criteria were enrolled in our study and divided into the training cohort (496 patients) and validation cohort (213 patients) (Figure 1). Clinicopathologic characteristics and imaging features in the training and validation cohorts are shown in Table 1. No differences were found between the two cohorts. HCC patients with histopathologically identified MVI accounted for $43.95 \%$ (218/496) and $41.31 \%(88 / 213)$ of the training and validation cohorts, respectively. There were no significant differences between them. Satisfactory interobserver and interobserver reproducibility of imaging features' extraction were achieved.

\section{Development of an MVI-predictive model}

A total of 34 variables (clinicopathologic characteristics and imaging features) were screened for the selection of most useful 8 potential predictors in the training cohort (Figure 2A,B), with non-zero coefficients in the LASSO and logistic regression model. Multi-category ordered variables were set as dummy variables. Results of univariate logistic analysis are showed in Table S2.

Independently associated risk factors of MVI were tumor diameter, ALP (>125 U/L), AFP (within $20-400$ or $\geq 400 \mathrm{ng} / \mathrm{mL}$ ), PIVKA-II (within $40-400$ or $\geq 400 \mathrm{mAU} / \mathrm{mL}$ ), multiple tumors, pseudo-capsule, infiltrative border with irregular shape, and intratumor inhomogeneous. A representative contrast-enhanced MRI image of 39-year-old HCC patients with MVI showed that pseudo-capsule manifested as a ring-shaped abnormal signal around the tumor in arterial phase (Figure $3 A$ ), portal venous phases (Figure 3B), and delay phases (Figure 3C). Pseudo-capsule was most obvious in delay phases.

Hemorrhagic or necrotic cystic signs inside the tumor (presented intratumor inhomogeneous) were also observed in arterial phase (Figure $3 D$ ), portal venous phases (Figure $3 E$ ), and delay phases (Figure $3 F$ ) of contrastenhanced MRI images from 47-year-old HCC patients with MVI. Infiltrative border with irregular shape of tumor was observed in T1 phase (Figure 3G), arterial phase (Figure 3H), portal venous phases (Figure 3I) from 53-year-old HCC patients with MVI.

The specific results on multivariate analysis are presented in Table 2. These candidate variables were incorporated to develop an MVI risk-predictive model, which was present 


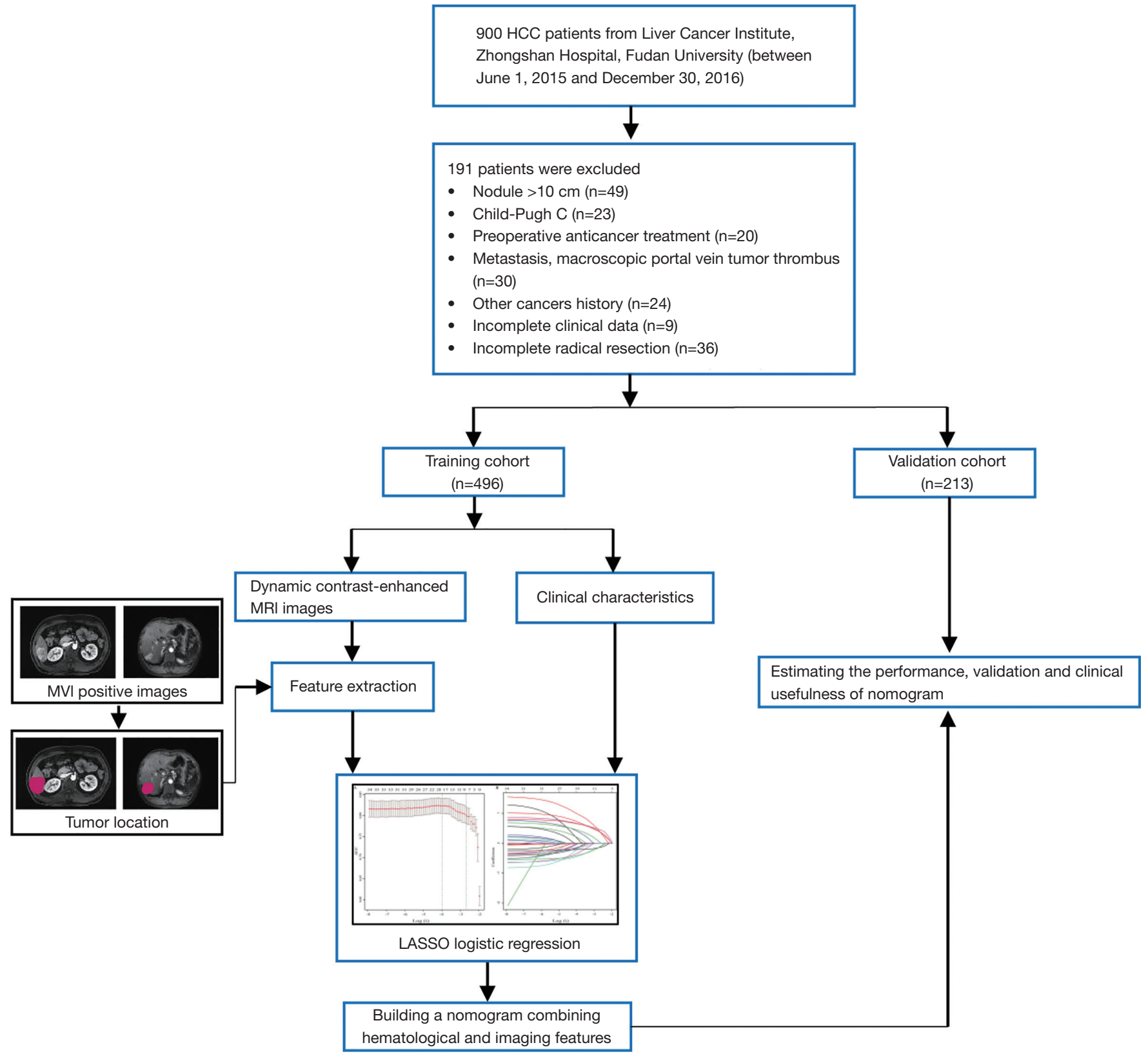

Figure 1 Flowchart of the study population and processing. HCC patients were categorized into two different groups (training, validation cohorts), and the further downstream processing was based on data from training cohort. HCC, hepatocellular carcinoma.

as the nomogram (Figure 4).

\section{Performance and validation of nomogram}

The predictive nomogram showed a good discrimination with a good C index of 0.82 (95\% CI, 0.782-0.857) (Figure $5 A$ ). The calibration plot for the MVI probability of the nomogram demonstrated good agreement between prediction and observation in the training cohort. The Hosmer-Lemeshow test $(\mathrm{P}=0.32)$ also showed that the model had a perfect fit relatively (Figure $5 B$ ). The favorable predictive accuracy of the nomogram was also confirmed in the validation cohort with a $\mathrm{C}$ index of $(0.80,95 \% \mathrm{CI}$, $0.772-0.837$ ) (Figure 5C). Moreover, good calibration of the 

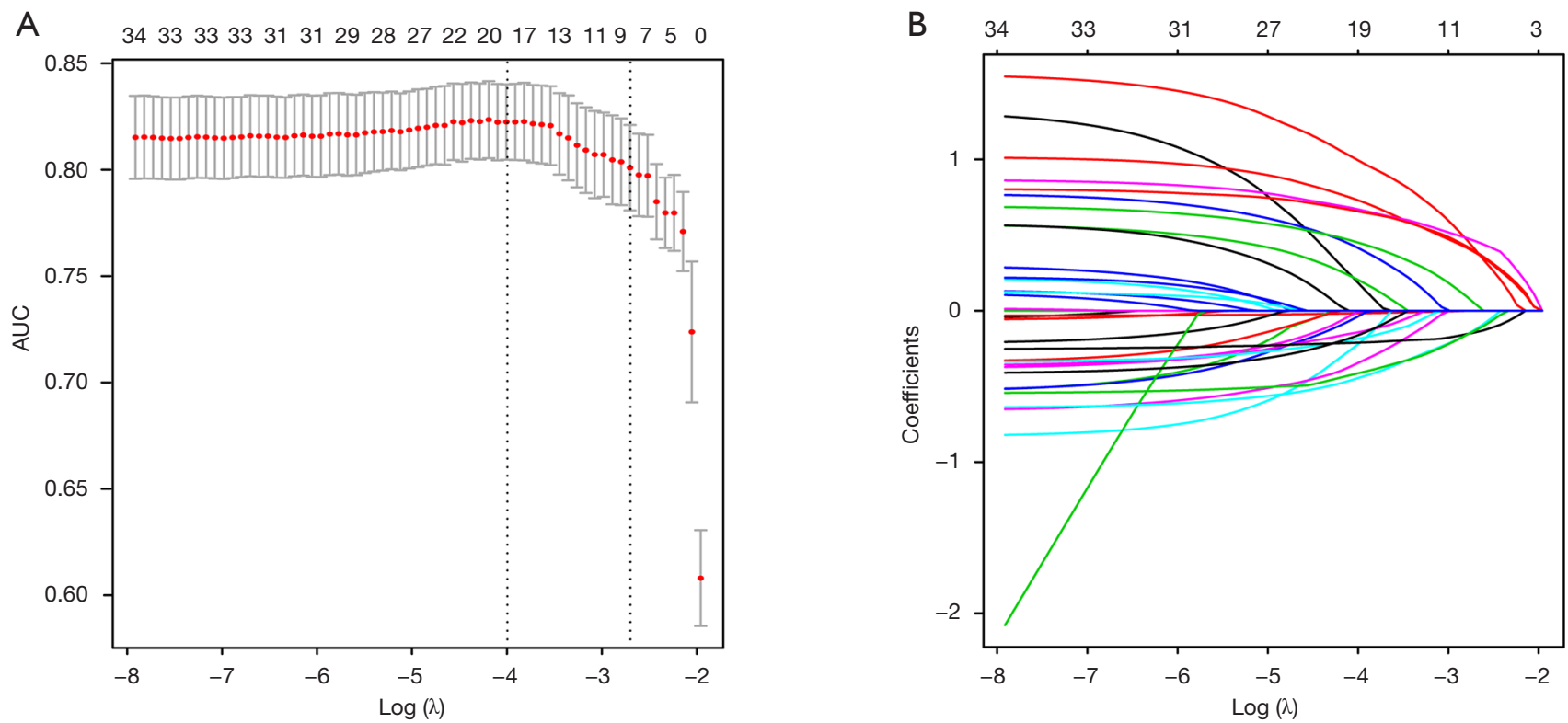

Figure 2 Feature selection using the LASSO binary logistic regression model. (A) Tuning parameter ( $\lambda$ ) was selected by LASSO model using 10 -fold cross-validation via minimum criteria. The vertical axis represents AUC which was plotted versus $\log (\lambda)$. The minimum criteria and the 1 standard error of the minimum criteria was chosen as the optimal values for dotted vertical lines drawn. A $\log (\lambda)$ value of -2.91 was chosen. (B) LASSO coefficient profiles of the 34 texture features produced based on $\log (\lambda)$ sequence. 10-fold cross-validation was used for optimal $\lambda$ resulting in 8 nonzero coefficients and vertical line drawn.

nomogram was also obtained for the probability of MVI in the validation cohort $(\mathrm{P}=0.31$, Hosmer Lemeshow test $)$ (Figure 5D).

The optimal cut-off value of the Nomo-score was 0.42 . The specificity and sensitivity of differentiating the presence or absence of MVI were $76.6 \%$ and $74.8 \%$ in the training cohort and $70.2 \%$ and $79.1 \%$ in the validation cohort, respectively. The performance of the nomogram (including optimal cut-off, sensitivity, specificity, positive predictive value, and negative predictive value) is summarized in Table 3.

\section{Clinical usefulness}

In order to estimate the clinical usefulness of the nomogram, we compared our model to other models of previous studies. Poté et al. found that AFP and PIVKAII could predict MVI (9). Other studies revealed that imaging features of contrast-enhanced MRI (including tumor diameter, tumor number, pseudo-capsules and arterial enhancement) were closely associated with MVI $(15,23)$. Therefore, a nomogram containing AFP, PIVKAII was defined as hematological test model. A nomogram containing imaging features of contrast-enhanced MRI was defined as imaging features' model. Our nomogram was defined as a combining model. The individual AUCs of the combined model, hematological test model and imaging features model were 82.0, 75.6, 74.5 and 80.5, 74.3, 71.9 in training cohort (Figure 6A) and validation cohort (Figure 6B) respectively. Delong test showed that the combining model's performance was better than the other two models $(\mathrm{P}<0.001)$. DCA was used to calculate net-benefits of our own nomogram to predict MVI at threshold probabilities of $40 \%$ and compare it to the other two predictive models mentioned above. Our model showed higher net-benefit than other predictive models (Figure 6C,D) and improved the reclassification performance in terms of NRI and IDI (Table 4).

\section{Effect of wide resection margin on the prognosis of MVI- positive HCC patients}

The surgical resection margin is an important prognostic factor of postoperative recurrence in hepatectomy for HCC patients. Negative surgical resection margin (R0) can reduce the postoperative recurrence rate and improve the 

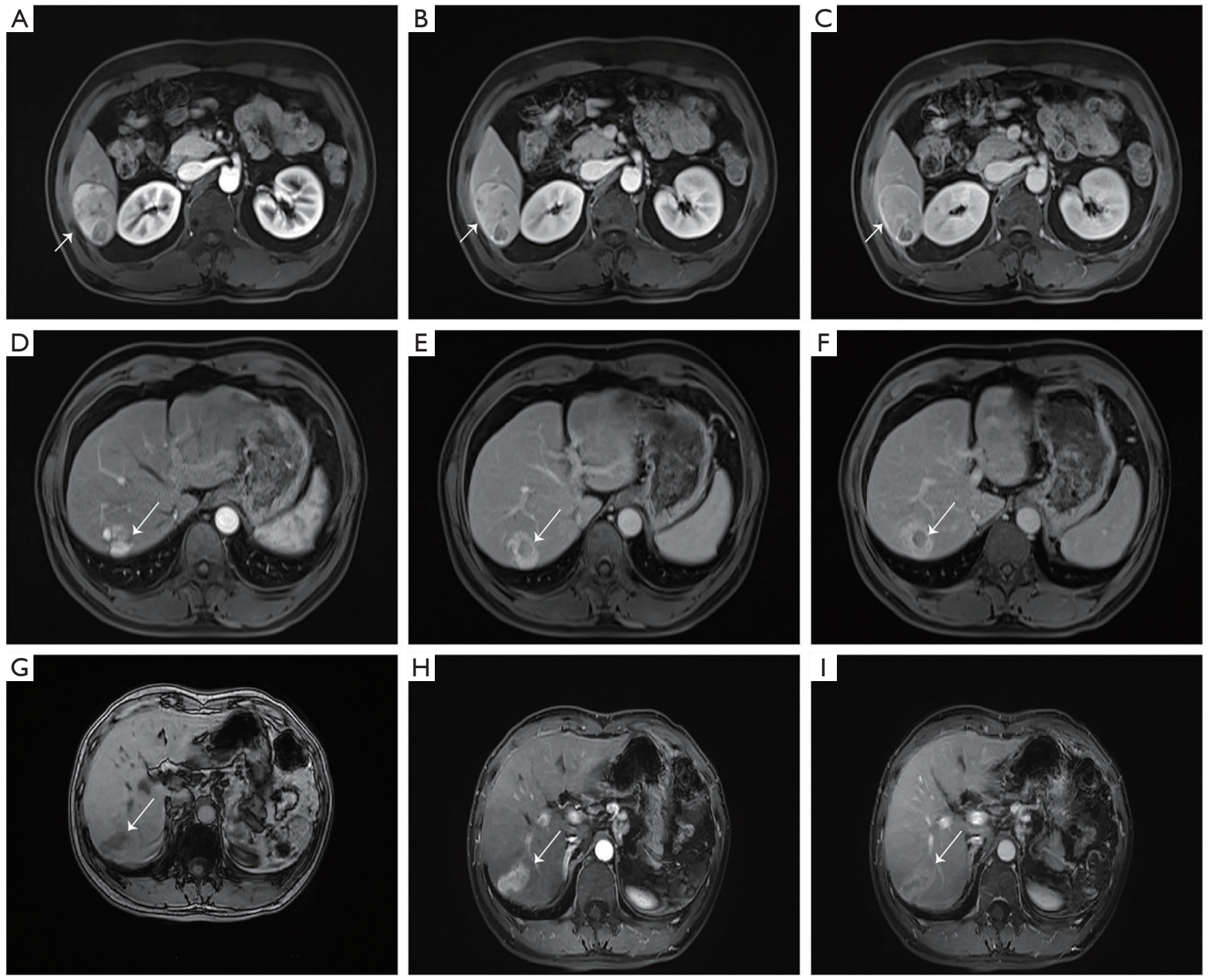

Figure 3 The representative images of three HCC patients with MVI (39-, 47- and 53-year-old respectively). Pseudo-capsule manifested as a ring-shaped abnormal signal around the tumor on arterial phase (A), portal venous phases (B), and delay phases (C). Hemorrhagic or necrotic cystic signs inside the tumor resulted in intratumor inhomogeneous on arterial phase (D), portal venous phases (E), and delay phases (F). Infiltrative border with irregular shape of tumor was observed in T1 phase (G), arterial phase (H), portal venous phases (I).

prognosis of patients with HCC. But the effect of resection margin width on recurrence-free and long-term survivals is still controversial. The width of resection margin more than $0.5 \mathrm{~cm}$ is recommended as beneficial. But recurrence after primary resection is multifactorial, $\mathrm{R} 0$ resection is just one of the problems in the treatment of HCC. MVI can invade the tumor capsule and blood vessels more than $1 \mathrm{~cm}$ away from the tumor edge. Therefore, we investigated the effect of resection with wide resection margin $(\geq 1 \mathrm{~cm})$ on the prognosis of MVI-positive HCC patients. After excluding patients with thin resection margin $(<0.5 \mathrm{~cm})$ and incomplete survival data a total of 650 patients were eligible. We then stratified patients according to tumor diameter $(<5$ and $\geq 5 \mathrm{~cm}$ ). In tumor diameter less than $5 \mathrm{~cm}$ and MVI-positive group, resection with wide margin $(\geq 1 \mathrm{~cm})$ had a better disease-free survival (DFS) compared to that with narrow margin $(0.5-1 \mathrm{~cm})$. In MVI-negative group, no statistically significant differences in DFS were found between the two groups (Figure $7 A, B$ ). The same conclusion was drawn from the group with tumor diameter 
Table 2 Multivariate logistic regression analysis of MVI presence in the training cohort

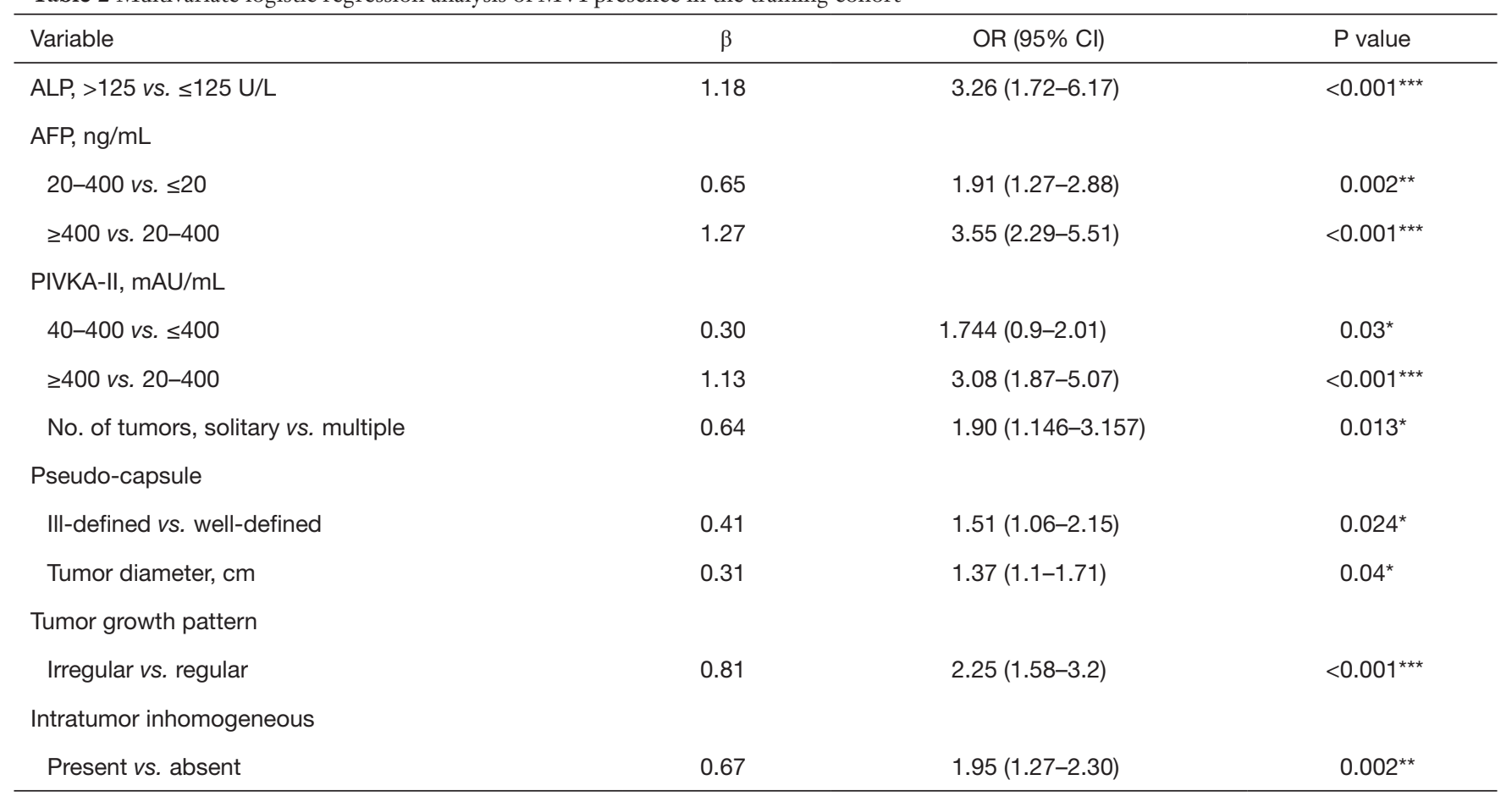

*, $\mathrm{P}<0.05 ;{ }^{* *}, \mathrm{P}<0.01 ;{ }^{* *}, \mathrm{P}<0.001 . \mathrm{MVI}$, microvascular invasion; ALP, alkaline phosphatase; AFP, alpha-fetoprotein; PIVKA-II, protein induced by vitamin $\mathrm{K}$ absence-II.

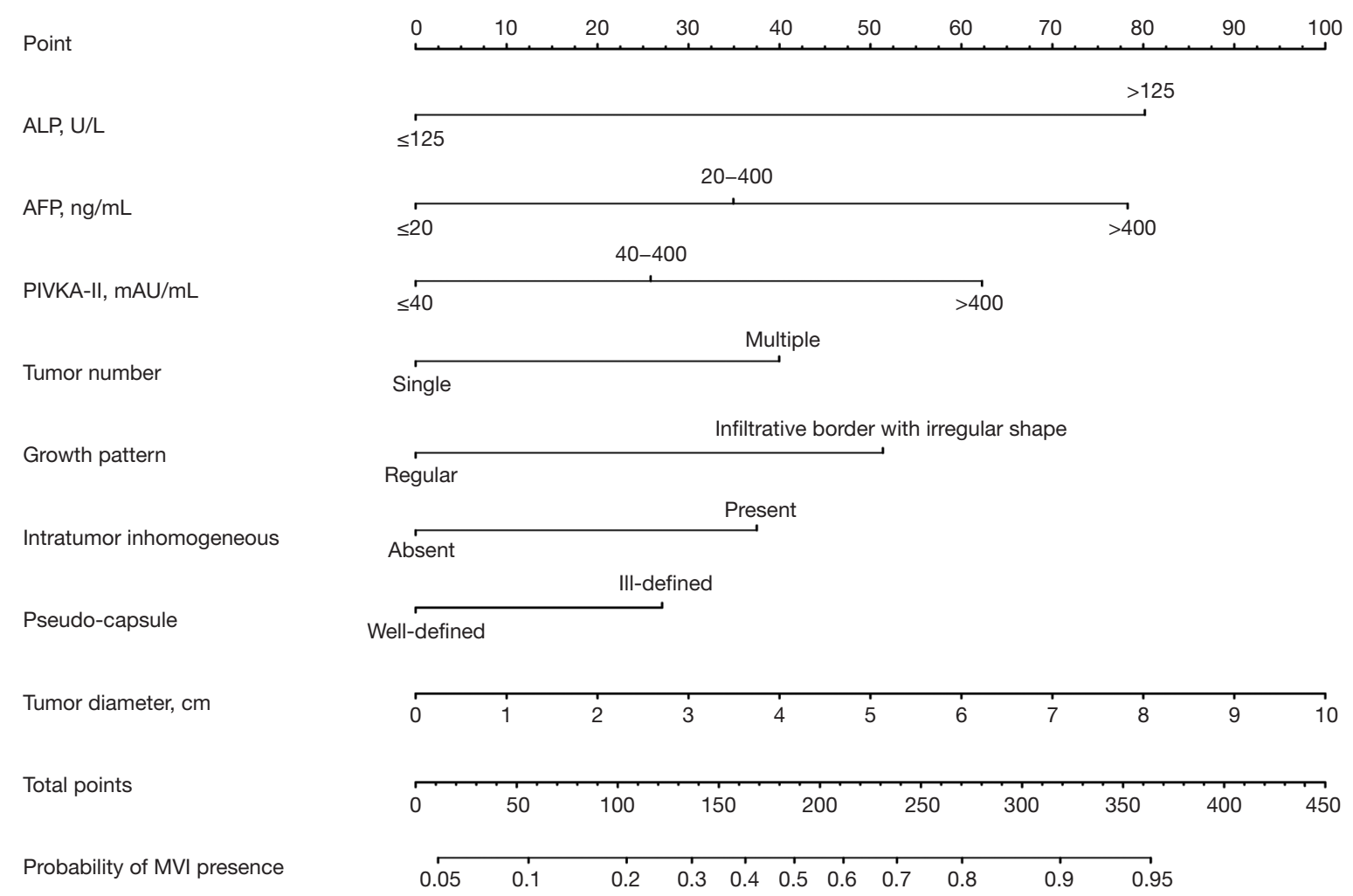

Figure 4 Nomogram for preoperative prediction of MVI. MVI, microvascular invasion. 
A

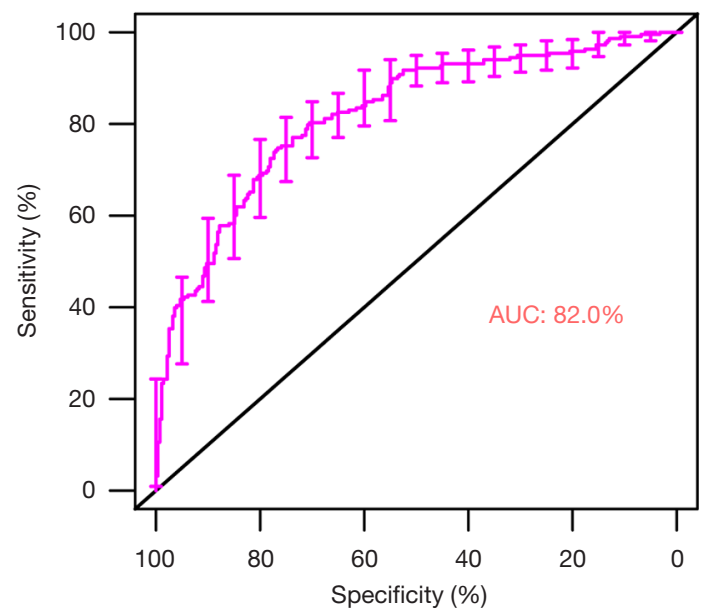

C

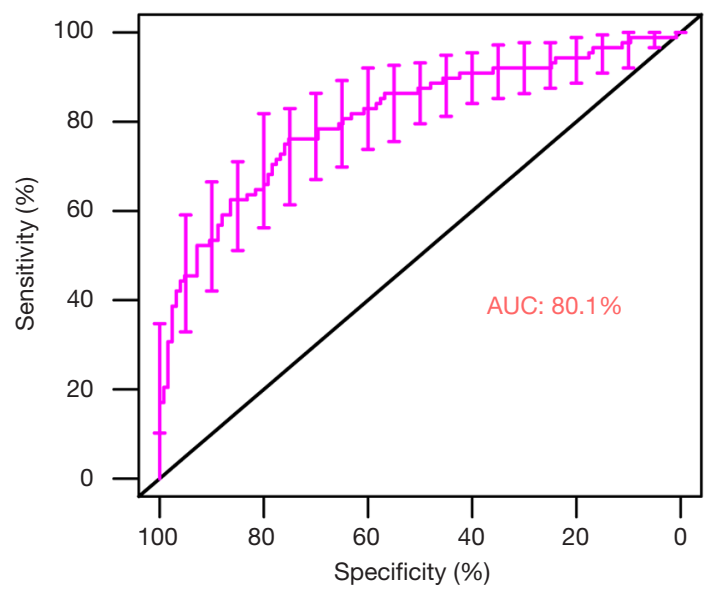

B Training cohort

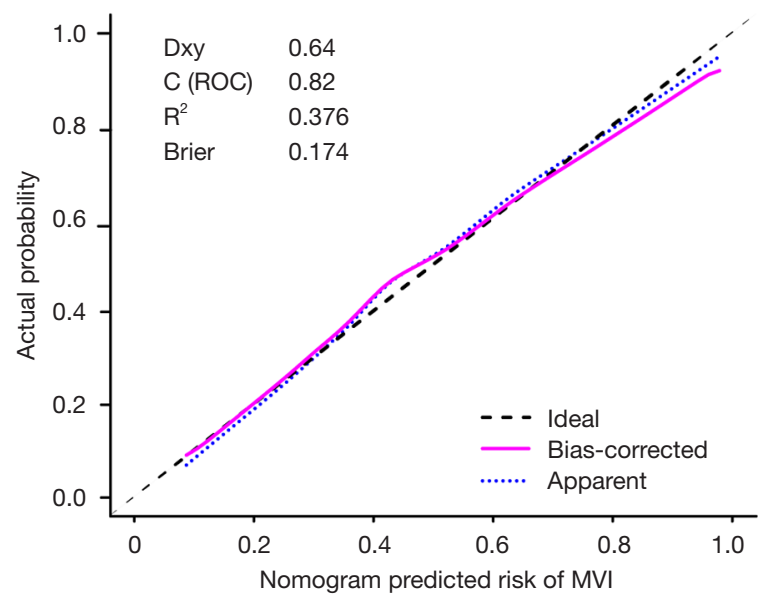

D

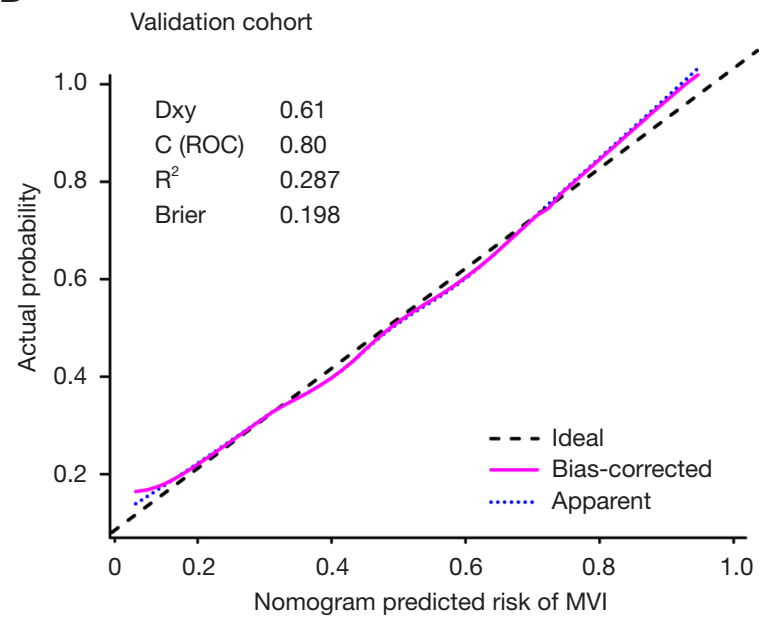

Figure 5 Diagnostic accuracy and Calibration curves of the nomogram for the estimation of MVI in HCC patients in the training and validation cohorts. In panel (A) and (C), a cut-off value of 0.4 of the Nom-score is used, ROC curves showed good diagnostic performance of the nomogram in the training and validation cohorts. In panel (B) and (D), Calibration curves of the nomogram in the training (B) and validation (D) cohorts are shown. Calibration curves depict the calibration of the model in terms of the agreement between the predicted probabilities of MVI presence and observed outcomes of MVI presence. The dotted black line represents an ideal prediction, and the dotted orange line represents the predictive ability of the nomogram, a closer fit to the diagonal dotted black line represents a better prediction. HCC, hepatocellular carcinoma; MVI, microvascular invasion.

more than $5 \mathrm{~cm}$ (Figure $7 C, D)$.

The nomogram also displayed the highest levels of accuracy to predict DFS in the training and validation cohorts. Other risk factors such as hepatoma cell differentiation and tumor diameter might affect the prognosis of HCC surgery. Therefore, a nomogram containing hepatoma cell differentiation and tumor diameter was defined as pathological features' model. DCA was used to calculate net-benefits of our own nomogram predicting DFS and compare it to pathological features' model and resection margin model (Figure S1). Our model showed higher net-benefit compared to other predictive models and improved the reclassification performance in terms of NRI and IDI (Table S3). Therefore, the prognostic 
Table 3 Performance of the prediction Nomo-score for estimating the risk of MVI

\begin{tabular}{lcc}
\hline \multirow{2}{*}{ Variable } & \multicolumn{2}{c}{ Value $(95 \% \mathrm{Cl})$} \\
\cline { 2 - 3 } Cutoff value & Training cohort & Validation cohort \\
AUC & 0.42 & 0.42 \\
Sensitivity, \% & $74.8(68.5-80.4)$ & $79.1(73.7-83.1)$ \\
Specificity, \% & $76.6(71.2-81.5)$ & $70.2(65.5-74.6)$ \\
$\begin{array}{l}\text { Positive predictive } \\
\text { value, \% }\end{array}$ & $71.5(65.2-77.3)$ & $66.8(61.6-71.6)$ \\
Negative predictive & $79.5(74.1-84.2)$ & $81.3(76.8-85.3)$ \\
value, \% & & $3.17(2.1-4.7)$ \\
$\begin{array}{l}\text { Positive likelihood } \\
\text { ratio }\end{array}$ & $3.20(2.55-4.01)$ & $0.36(0.26-0.49)$ \\
Negative likelihood & $0.32(0.26-0.41)$ & 74.04 \\
ratio & & 75.8 \\
Accuracy & & \\
\hline
\end{tabular}

MVI, microvascular invasion; AUC, area under curve.

nomogram was also distinctly valuable in recurrence prediction.

\section{Discussion}

MVI is a well-known risk factor that affects the recurrence of HCC and survival of patients after undergoing hepatectomy. In the present study, approximately $40 \%$ of HCC patients harbored MVI. Therefore, it is necessary to establish an efficient predictive model preoperatively. We developed and validated a diagnostic and practical predictive model for preoperative MVI prediction that combines hematological test (including inflammatory and tumor markers of HCC) and imaging features of contrastenhanced MRI.

For the construction of the nomogram, a total of 34 clinicopathological characteristics and imaging features were selected and combined into a marker panel by LASSO method. The nomogram incorporated 8 comprehensive and accessible preoperative variables [including tumor diameter, ALP (>125 U/L), AFP (within 20-400 or $\geq 400 \mathrm{ng} / \mathrm{mL}$ ], PIVKA-II (within $40-400$ or $\geq 400 \mathrm{mAU} / \mathrm{mL}$ ), multiple tumors, pseudo-capsule, infiltrative border with irregular shape, and intratumor inhomogeneous). Moreover, it performed well with a good discrimination and $\mathrm{C}$ index of 0.82 (95\% CI, 0.782-0.857) and 0.80, (95\%
CI, 0.772-0.837) in the training and validation cohorts, respectively. The calibration plot for the MVI probability of the nomogram demonstrated good agreement between prediction and observation.

A variety of previous studies tried to found effective preoperative variables to predict MVI. Poté et al. found that PIVKA-II was associated with MVI, but he did not build a predictive model for clinical applications (9). Hyun et al. also built an improved predictive model combining standardized uptake value ratio on FDG PET/CT, tumor size, and AFP, but the performance of this model (AUC $=0.756$ ) is not as well as ours and PET/CT was not extensively used (24). In other studies, radiomics-score of imaging features based on contrast-enhanced CT, gadoxetic acid-enhanced MRI, and ultrasound were extensively used to build nomograms. However, these thousands of radiomic features could only be obtained by specific computers and software (4,5,25-28). Therefore, they were not practical for surgeons' use. Lei et al. also built an MVI-predictive model for patients with HBV-related HCC (29). However, $\mathrm{HBV}$-related HCC is mainly common in China, while our model is applicable for the MVI prediction of HCC of all different etiologies. In the present study, hematological tests including inflammatory and tumor markers of HCC and imaging features of contrast-enhanced MRI are all accessible, and the nomogram has high accuracy, good discrimination and high net-benefit for surgeons' use.

In a multicenter clinical trial containing 1,109 patients with solitary HCC, Shindoh et al. found that MVI presence was not associated with poor prognosis of small HCC $(\leq 2 \mathrm{~cm})(30)$. Thus, the relation between presence of MVI and prognosis of HCC is still controversial. Therefore, tumors with diameters larger than $5 \mathrm{~cm}$ were included in our study. Presence of MVI in advanced HCC is common, so we excluded cases with tumor diameter larger than 10 $\mathrm{cm}$ (31). Tumor diameter was an independent risk factor of MVI in our study. Surgeons can choose suitable resection margin based on the nomogram score.

Recently, PIVKA-II has been adopted as biomarker for the early detection of HCC. Previous studies found that PIVKA-II can improve angiogenesis around HCC tissues (32). Fujikawa et al. used human umbilical vein endothelial cells (HUVEC) to analyze the angiogenic effects of PIVKA-II (33). The results showed that PIVKA-II can promote DNA synthesis and HUVEC migration. Wang et al. found that PIVKA-II can promote the expression of various angiogenic factors in HCC cells, including vascular endothelial growth 
A

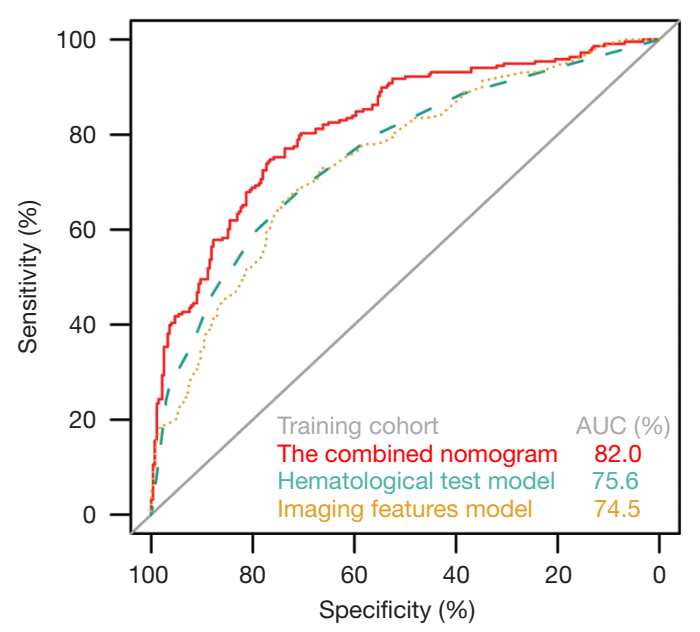

C Training cohort

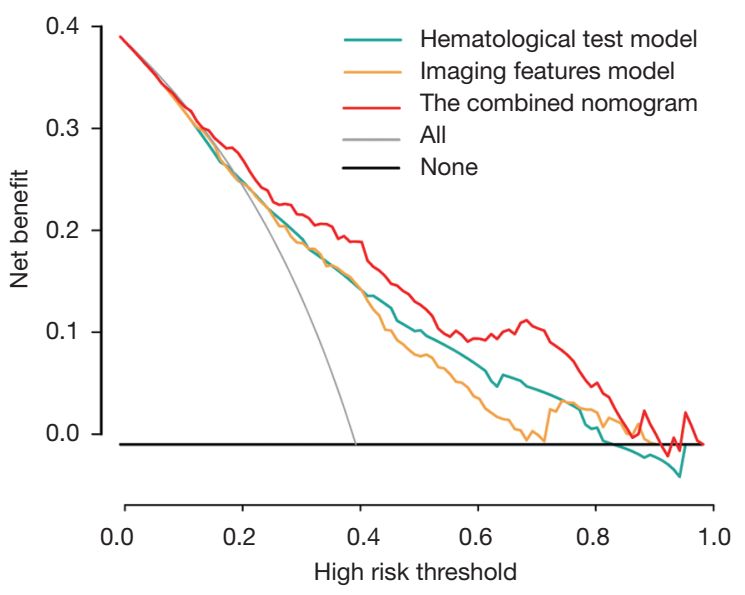

B

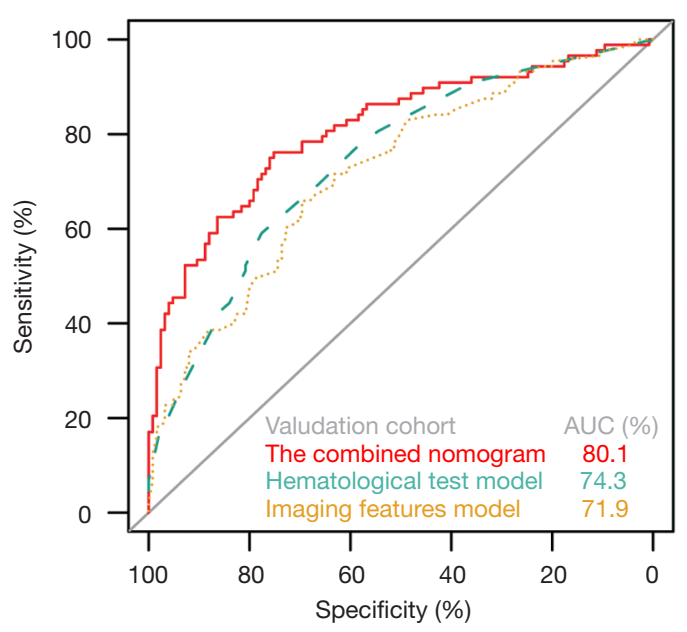

D Validation cohort

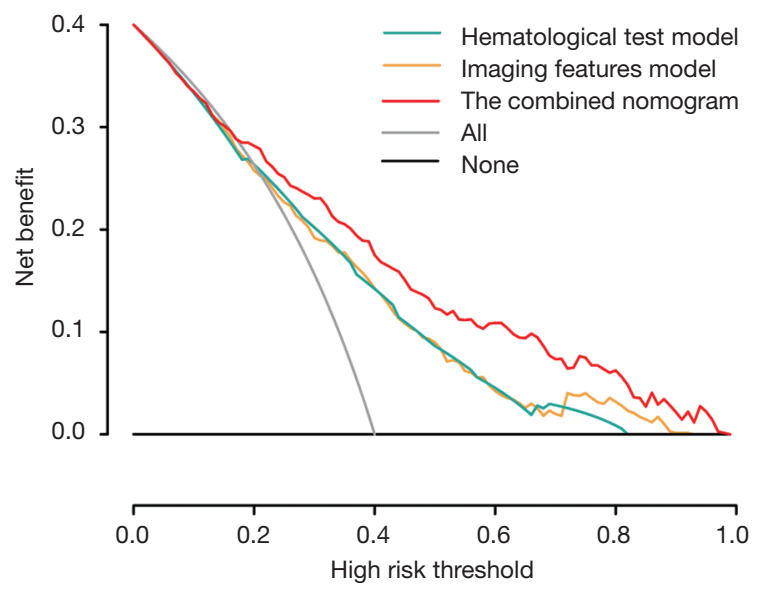

Figure 6 The ROC curve of the prognostic nomogram, hematological test model, imaging features model and combined model in the training (A) and validation cohorts (B). Decision curve analysis (DCA) of each model in predicting MVI for HCC patients. The vertical axis measures standardized net-benefit. The horizontal axis shows the corresponding risk threshold. The DCA shows that if the threshold probability is between 0 and 0.8 , using the present nomogram derived from this study (red curve) to predict MVI presence provides a greater benefit than the hematological test model (green curve) and Imaging features alone (orange curve) in previous studies. Notes: The DCA curve for training cohort $(\mathrm{C})$ and validation cohort $(\mathrm{D})$ are also shown.

factor (VEGF), transforming growth factor- $\beta$ (TGF- $\beta$ ), and basic fibroblast growth factor (bFGF) (34). The above research results suggested that PIVKA-II secreted by liver cancer may induce angiogenesis in tissues surrounding liver cancer, and then affect the prognosis of patients. Poté et al. found that PIVKA-II can predict MVI (9). In our study, we chose 40 and $400 \mathrm{mAU} / \mathrm{mL}$ as the split points to divide PIVKA-II for three levels, based on previous studies. The results showed that PIVKA-II more than $400 \mathrm{mAU} / \mathrm{mL}$ yielded a high risk of MVI.

ALP exists in various tissues, and is more abundant in liver and bone. ALP expression increases significantly in cholestatic and hepatobiliary diseases. Wang et al. uncovered that high ALP was an independent risk factors 


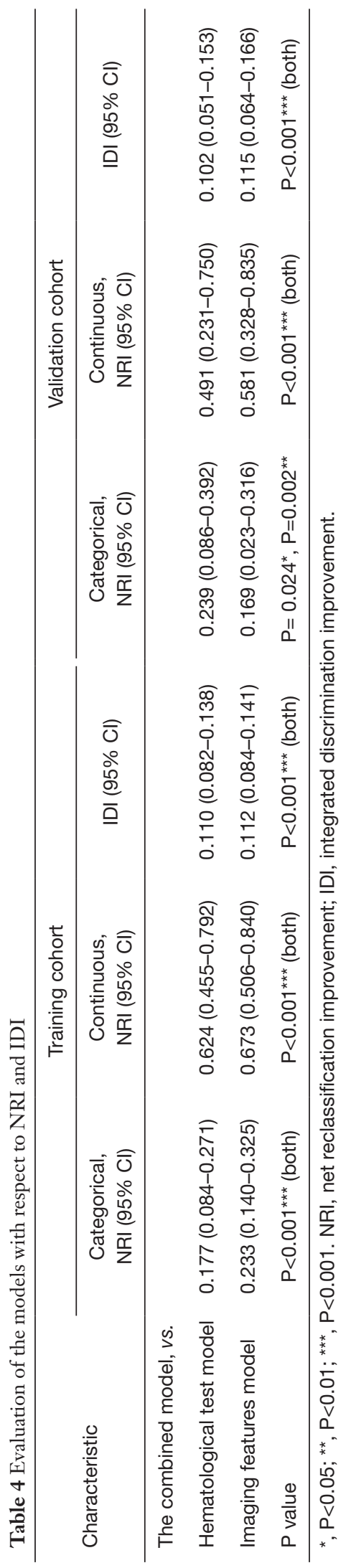

for poor overall survival (OS) (35). Du et al. also found that serum ALP level in HCC patients was a predictive indicator of bone metastasis (36). However, the mechanism was not very clear. One study presented that ALP and ALP-tolymphocyte ratio (ALR) count were independent predictors of MVI (37). In the present study, we also found that abnormal ALP values contributed greatly to the occurrence of MVI.

Four significant imaging features (tumor diameter, number, infiltrative border with irregular shape and pseudo-capsules) that reflect tumor aggressiveness were also included in our model. The expansive growth of tumor results in mechanical compression of the adjacent liver parenchyma leading to ischemic, necrotic damage, and capsule formation. Thus, tumors with infiltrative growth promote capsule formation (38). This may be due to the uneven distribution of tumor neovascularization or the complexity of tumor components, such as tumor bleeding, necrosis, or fat-containing ingredients such as iron. Intratumor inhomogeneous was also included in our model.

This model has good clinical applicability. The optimal cut-off value of the Nomo-score was determined to be 0.42 . The specificity, and sensitivity of differentiating the presence or absence of MVI were $87.3 \%$ and $72.2 \%$, respectively. DCA showed that our model had higher netbenefit compared to other predictive models based only on hematological or imaging features. It also improved the reclassification performance.

Although some studies showed that anatomical resection did not promote the prognosis, the mainstream view is that anatomical liver resection is better than local resection, which can reduce intrahepatic metastasis of HCC through portal veins (39-41). MVI is considered to be the initial form of HCC metastasis through the portal vein, and an important sign of HCC aggressiveness and poor prognosis $(42,43)$. Our result showed that compared to patients with narrow resection margin $(0.5-1 \mathrm{~cm})$, those with wide resection margin $(>1 \mathrm{~cm})$ had a significantly lower 3-year recurrence rate. That suggests that resection with wide margin for the treatment of MVI-positive HCC patients has a better DFS. MVI is a significant factor that affects the recurrence of HCC after hepatectomy. Thus, we chose to analyze the effect of resection with wide margin $(\geq 1 \mathrm{~cm})$ on the DFS of MVI-positive HCC patients rather than OS. Due to liver background lesions and tumor location, surgeons often encounter situations where anatomical resection cannot be completed or only completed with thin resection margin. The result of this study can help 
A
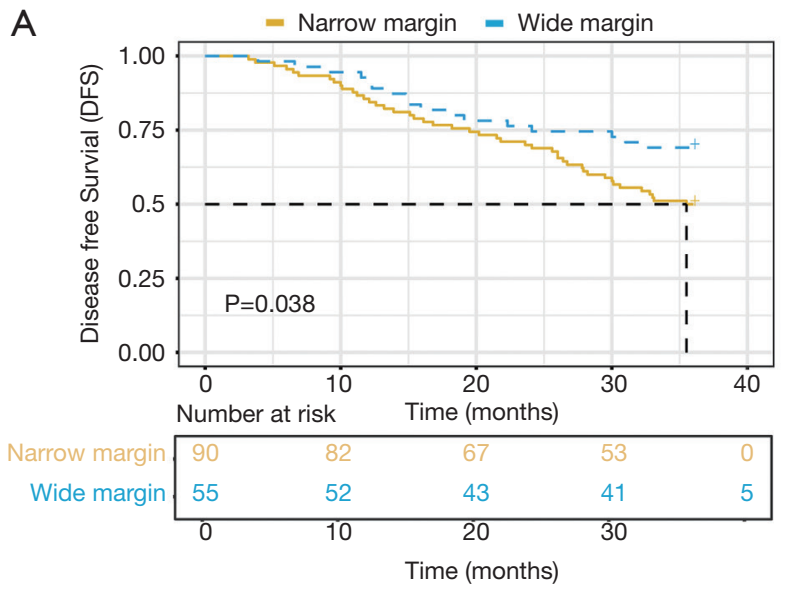

C

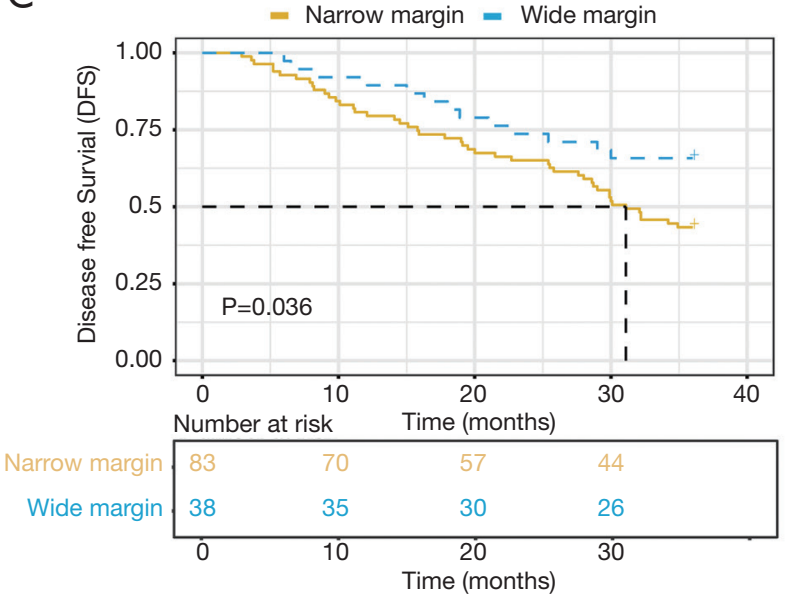

B

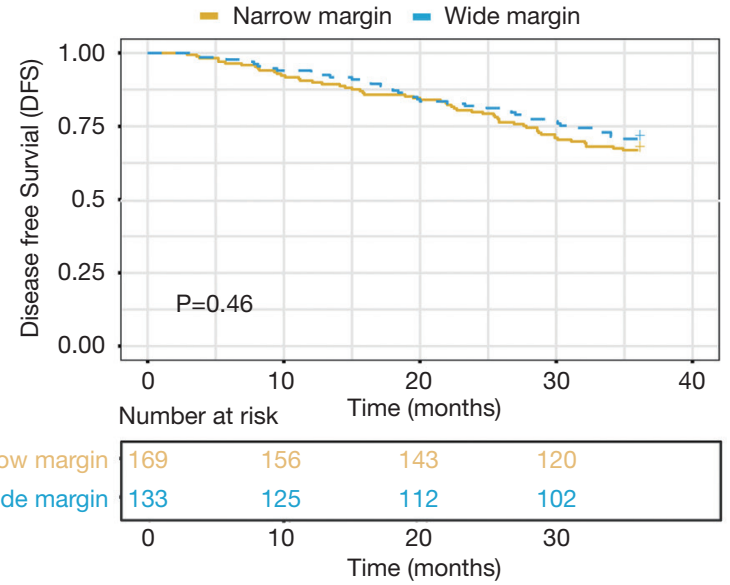

D

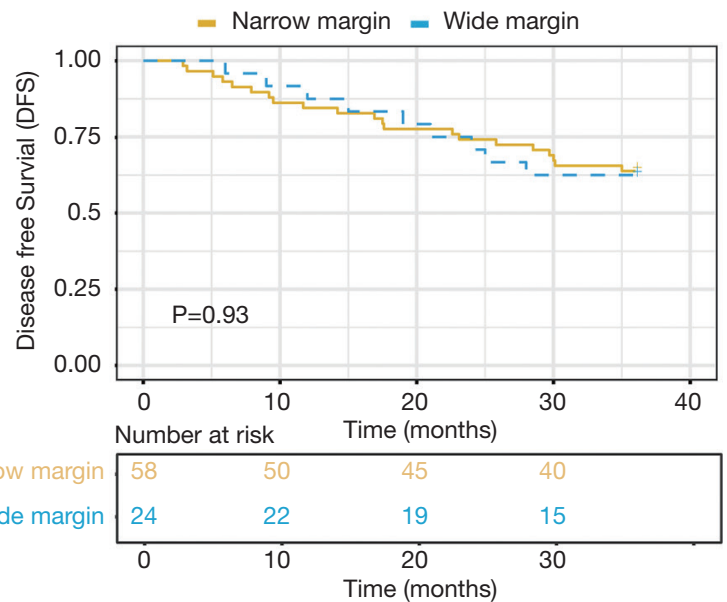

Figure 7 Effect of wide resection margin on the prognosis of MVI-positive HCC patients. In MVI-positive HCC patients with tumor diameter less than $5 \mathrm{~cm}$, it showed that resection with wide margin $(\geq 1 \mathrm{~cm})$ of HCC patients had a better DFS than those with narrow margin $(0.5-1 \mathrm{~cm})(\mathrm{A})$. In MVI-negative group, there are not statistically significant differences in DFS between the two group (B). The same conclusion was drawn from the other group with tumor diameter more than $5 \mathrm{~cm}(\mathrm{C}, \mathrm{D})$.

surgeons choose an appropriate treatment strategy such as neoadjuvant therapy for HCC patients.

Some limitations in our study should be noted. First, potential selection bias may impact the reliability and reproducibility of the model because the trial was carried retrospectively. Secondly, this study was based on singlecenter experience. Thus, further clinical studies are needed to evaluate its accuracy. Thirdly, in contrast to previous reports, ALP, PIVKA-II (within 40-400 or $\geq 400 \mathrm{mAU} / \mathrm{mL}$ ), intratumor inhomogeneous imaging or with necrosis and hemorrhage were included in our model. Therefore, we need more external validation cohort to validate our model.

\section{Conclusions}

This study developed and validated a diagnostic and practical preoperative predictive model of MVI that combined hematological test and imaging features of contrast-enhanced MRI [including tumor diameter, ALP (>125 U/L), AFP (within 20-400 or $\geq 400 \mathrm{ng} / \mathrm{mL}$ ), PIVKAII (within $40-400$ or $\geq 400 \mathrm{mAU} / \mathrm{mL}$ ), multiple tumors, pseudo-capsule, infiltrative border with irregular shape, and intratumor inhomogeneous]. The present nomogram performed well in discrimination and accuracy of MVI prediction in both training and validation cohorts and showed higher net-benefit compared to other predictive 
models based on only hematological or imaging features. It also improved the reclassification performance. This study also found that resection with wide margin for the treatment of MVI-positive HCC patients had a better DFS. Therefore, the model can help surgeons decide if a wide resection margin is needed based on the nomogram score and, therefore, choose an appropriate treatment strategy such as neoadjuvant therapy or molecular targeted therapy for advanced HCC stages.

\section{Acknowledgments}

We thank the patients who provided consent for the use of their clinical data knowing that they may not directly derive benefit.

Funding: This work was supported by grants from the Shanghai International Science and Technology Collaboration Program (18410721900), the National Natural Science Foundation of China (81472672), and Shanghai Municipal Key Clinical Specialty. The Science and Technology Plan Project of Xiamen City (3502Z20194036). Research Incubation Project of Xiamen Branch, Zhongshan Hospital, Fudan University (2018ZSXMWK01).

\section{Footnote}

Reporting Checklist: The authors have completed the TRIPOD reporting checklist. Available at http://dx.doi. org/10.21037/atm-20-4695

Data Sharing Statement: The data that support the findings of this study are available from Ning Ren upon reasonable request. Available at http://dx.doi.org/10.21037/atm-204695

Conflicts of Interest: All authors have completed the ICMJE uniform disclosure form (available at http://dx.doi. org/10.21037/atm-20-4695). The authors have no conflicts of interest to declare.

Ethical Statement: The authors are accountable for all aspects of the work in ensuring that questions related to the accuracy or integrity of any part of the work are appropriately investigated and resolved. The study was conducted in accordance with the Declaration of Helsinki (as revised in 2013). Clinical data in the study was obtained from Zhongshan hospital medical system. The study was approved by Ethics Committee of Zhongshan Hospital,
Fudan University. Approval No. is Y2018-155. Informed consent of clinical data used in this study was obtained from all patients without financial compensation.

Open Access Statement: This is an Open Access article distributed in accordance with the Creative Commons Attribution-NonCommercial-NoDerivs 4.0 International License (CC BY-NC-ND 4.0), which permits the noncommercial replication and distribution of the article with the strict proviso that no changes or edits are made and the original work is properly cited (including links to both the formal publication through the relevant DOI and the license). See: https://creativecommons.org/licenses/by-nc-nd/4.0/.

\section{References}

1. Siegel RL, Miller KD, Jemal A. Cancer statistics, 2020. CA Cancer J Clin 2020;70:7-30.

2. Gluer AM, Cocco N, Laurence JM, et al. Systematic review of actual 10-year survival following resection for hepatocellular carcinoma. HPB (Oxford) 2012;14:285-90.

3. Roayaie S, Blume IN, Thung SN, et al. A system of classifying microvascular invasion to predict outcome after resection in patients with hepatocellular carcinoma. Gastroenterology 2009;137:850-5.

4. Xu X, Zhang HL, Liu QP, et al. Radiomic analysis of contrast-enhanced CT predicts microvascular invasion and outcome in hepatocellular carcinoma. J Hepatol 2019;70:1133-44.

5. Lee S, Kim SH, Lee JE, et al. Preoperative gadoxetic acidenhanced MRI for predicting microvascular invasion in patients with single hepatocellular carcinoma. J Hepatol 2017;67:526-34.

6. Wang WT, Yang L, Yang ZX, et al. Assessment of Microvascular Invasion of Hepatocellular Carcinoma with Diffusion Kurtosis Imaging. Radiology 2018;286:571-80.

7. Banerjee S, Wang DS, Kim HJ, et al. A computed tomography radiogenomic biomarker predicts microvascular invasion and clinical outcomes in hepatocellular carcinoma. Hepatology 2015;62:792-800.

8. Lok AS, Sterling RK, Everhart JE, et al. Des-gammacarboxy prothrombin and alpha-fetoprotein as biomarkers for the early detection of hepatocellular carcinoma. Gastroenterology 2010;138:493-502.

9. Poté N, Cauchy F, Albuquerque M, et al. Performance of PIVKA-II for early hepatocellular carcinoma diagnosis and prediction of microvascular invasion. J Hepatol 2015;62:848-54. 
10. Dahiya D, Wu TJ, Lee CF, et al. Minor versus major hepatic resection for small hepatocellular carcinoma (HCC) in cirrhotic patients: a 20-year experience. Surgery 2010;147:676-85.

11. Dong S, Wang Z, Wu L, et al. Effect of surgical margin in R0 hepatectomy on recurrence-free survival of patients with solitary hepatocellular carcinomas without macroscopic vascular invasion. Medicine 2016;95:e5251.

12. Oguro S, Yoshimoto J, Imamura H, et al. Clinical significance of macroscopic no-margin hepatectomy for hepatocellular carcinoma. HPB (Oxford) 2018;20:872-80.

13. Rajakannu M, Cherqui D, Ciacio O, et al. Liver stiffness measurement by transient elastography predicts late posthepatectomy outcomes in patients undergoing resection for hepatocellular carcinoma. Surgery 2017;162:766-74.

14. Shen Y, Zhou C, Zhu G, et al. Liver Stiffness Assessed by Shear Wave Elastography Predicts Postoperative Liver Failure in Patients with Hepatocellular Carcinoma. J Gastrointest Surg 2017;21:1471-9.

15. Kim MJ, Lee M, Choi JY, et al. Imaging features of small hepatocellular carcinomas with microvascular invasion on gadoxetic acid-enhanced MR imaging. Eur J Radiol 2012;81:2507-12.

16. Choi YS, Rhee H, Choi JY, et al. Histological characteristics of small hepatocellular carcinomas showing atypical enhancement patterns on gadoxetic acid-enhanced MR imaging. J Magn Reson Imaging 2013;37:1384-91.

17. Bruix J, Sherman M; Practice Guidelines Committee, American Association for the Study of Liver Diseases. Management of hepatocellular carcinoma. Hepatology 2005;42:1208-36.

18. Marrero JA, Feng Z, Wang Y, et al. Alpha-fetoprotein, des-gamma carboxyprothrombin, and lectin-bound alpha-fetoprotein in early hepatocellular carcinoma. Gastroenterology 2009;137:110-8.

19. Wang Y, Li J, Xia Y, et al. Prognostic nomogram for intrahepatic cholangiocarcinoma after partial hepatectomy. J Clin Oncol 2013;31:1188-95.

20. Shariat SF, Karakiewicz PI, Suardi N, et al. Comparison of nomograms with other methods for predicting outcomes in prostate cancer: a critical analysis of the literature. Clin Cancer Res 2008;14:4400-7.

21. Harrell FE Jr, Califf RM, Pryor DB, et al. Evaluating the yield of medical tests. JAMA 1982;247:2543-6.

22. Fitzgerald M, Saville BR, Lewis RJ. Decision curve analysis. JAMA 2015;313:409-10.

23. Witjes CD, Willemssen FE, Verheij J, et al. Histological differentiation grade and microvascular invasion of hepatocellular carcinoma predicted by dynamic contrastenhanced MRI. J Magn Reson Imaging 2012;36:641-7.

24. Hyun SH, Eo JS, Song BI, et al. Preoperative prediction of microvascular invasion of hepatocellular carcinoma using 18F-FDG PET/CT: a multicenter retrospective cohort study. Eur J Nucl Med Mol Imaging 2018;45:720-6.

25. Ma X, Wei J, Gu D, et al. Preoperative radiomics nomogram for microvascular invasion prediction in hepatocellular carcinoma using contrast-enhanced CT. Eur Radiol 2019;29:3595-605.

26. Hu HT, Wang Z, Huang XW, et al. Ultrasound-based radiomics score: a potential biomarker for the prediction of microvascular invasion in hepatocellular carcinoma. Eur Radiol 2019;29:2890-901.

27. Xiachuan Q, Xiang Z, Xuebing L, et al. Predictive Value of Contrast-enhanced Ultrasound for Early Recurrence of Single Lesion Hepatocellular Carcinoma After Curative Resection. Ultrason Imaging 2019;41:49-58.

28. Yang L, Gu D, Wei J, et al. A Radiomics Nomogram for Preoperative Prediction of Microvascular Invasion in Hepatocellular Carcinoma. Liver Cancer 2019;8:373-86.

29. Lei Z, Li J, Wu D, et al. Nomogram for Preoperative Estimation of Microvascular Invasion Risk in Hepatitis B Virus-Related Hepatocellular Carcinoma Within the Milan Criteria. JAMA Surg 2016;151:356-63.

30. Shindoh J, Andreou A, Aloia TA, et al, Microvascular invasion does not predict long-term survival in hepatocellular carcinoma up to $2 \mathrm{~cm}$. reappraisal of the staging system for solitary tumors. Ann Surg Oncol 2013;20:1223-9.

31. Rodríguez-Perálvarez M, Luong TV, Andreana L, et al. A systematic review of microvascular invasion in hepatocellular carcinoma: diagnostic and prognostic variability. Ann Surg Oncol 2013;20:325-39.

32. Zhou C, Chen W, Sun J, et al. Low expression of WW domain-containing oxidoreductase associates with hepatocellular carcinoma aggressiveness and recurrence after curative resection. Cancer Med 2018;7:3031-43.

33. Fujikawa T, Shiraha H, Ueda N, et al. Des-gammacarboxyl prothrombin-promoted vascular endothelial cell proliferation and migration. J Biol Chem 2007;282:8741-8.

34. Wang SB, Cheng YN, Cui SX, et al. Des-gamma-carboxy prothrombin stimulates human vascular endothelial cell growth and migration. Clin Exp Metastasis 2009;26:469-77.

35. Wang H, Du PC, Wu MC, et al. Postoperative adjuvant transarterial chemoembolization for multinodular hepatocellular carcinoma within the Barcelona Clinic 
Liver Cancer early stage and microvascular invasion. Hepatobiliary Surg Nutr 2018;7:418-28.

36. Du WX, Duan SF, Chen JJ, et al. Serum bone-specific alkaline phosphatase as a biomarker for osseous metastases in patients with malignant carcinomas: a systematic review and meta-analysis. J Cancer Res Ther 2014;10 Suppl:C140-3.

37. Zhu Y, Xu D, Zhang Z, et al. A new laboratory-based algorithm to predict microvascular invasion and survival in patients with hepatocellular carcinoma. Int J Surg 2018;57:45-53.

38. Grigioni WF, D'Errico A, Biagini G, et al. The capsule surrounding primary liver tumors: wherefrom its prognostic significance? Int J Cancer 1990;45:637-43.

39. Marubashi S, Gotoh K, Akita H, et al. Analysis of Recurrence Patterns After Anatomical or Non-anatomical Resection for Hepatocellular Carcinoma. Ann Surg Oncol 2015;22:2243-52.

Cite this article as: Zhou Q, Zhou C, Yin Y, Chen W, Liu C, Atyah M, Weng J, Shen Y, Yi Y, Ren N. Development and validation of a nomogram combining hematological and imaging features for preoperative prediction of microvascular invasion in hepatocellular carcinoma patients. Ann Transl Med 2021;9(5):402. doi: 10.21037/atm-20-4695
40. Shin S, Kim TS, Lee JW, et al. Is the anatomical resection necessary for single hepatocellular carcinoma smaller than $3 \mathrm{~cm}$ ?: single-center experience of liver resection for a small HCC. Ann Hepatobiliary Pancreat Surg 2018;22:326-34.

41. Moris D, Tsilimigras DI, Kostakis ID, et al. Anatomic versus non-anatomic resection for hepatocellular carcinoma: A systematic review and meta-analysis. Eur J Surg Oncol 2018;44:927-38.

42. Erstad DJ, Tanabe KK. Prognostic and Therapeutic Implications of Microvascular Invasion in Hepatocellular Carcinoma. Ann Surg Oncol 2019;26:1474-93.

43. Subbotin VM. Privileged portal metastasis of hepatocellular carcinoma in light of the coevolution of a visceral portal system and liver in the chordate lineage: a search for therapeutic targets. Drug Discov Today 2018;23:548-64. 


\section{Appendix 1 Detailed descriptions of the statistical methodology}

LASSO and 10-fold cross-validation was used to select and sort the statistically significant MVI related features in the training cohort. A log partial likelihood minimization was carried out under the constraint of the sum of the absolute values of the parameters multiplied by a constant (44).

$\hat{\beta}=\arg \min l(\beta)$, subject to $\sum\left|\beta_{j}\right| \leq t$

where $\hat{\beta}$ is parameter obtained through LASSO algorithm, $l(\beta)$ denotes the log-partial likelihood in the logistic regression model, and $t>0$ represents a constant. The LASSO algorithm automatically selects variables and shrinks some coefficients and reduces others to exactly 0 via the absolute constraint. In this study, the constant t was set at 0.01 , and 8 nonzero coefficients were selected through LASSO algorithm for the present model (45).

\section{Appendix 2 Analysis of DCA}

The DCA was conducted to evaluate the clinical utility of this nomogram. The clinical utility of different MVI prediction models mentioned in this manuscript were compared and assessed through DCA after analyzing the net benefits within the range of threshold probabilities (46). The theory and computational method of the DCA is illustrated with the following equation:

$$
\frac{a-c}{d-b}=\frac{1-P t}{P t}
$$

When comparing two models for predicting or diagnosing a disease, $b$ indicates net benefit from treated patients based on the model and $\mathrm{d}$ indicates net benefit from treating all patients. $a-c$ represents net benefit for the untreated, which also can be interpreted as number of avoidable treatments. $P t$ is the threshold probability.

\section{References}

44. Sauerbrei W, Royston P, Binder H. Selection of important variables and determination of functional form for continuous predictors in multivariable model building. Stat Med 2007;26:5512-28.

45. Jiang M, Li C, Tang S, et al. Nomogram Based on ShearWave Elastography Radiomics Can Improve Preoperative Cervical Lymph Node Staging for Papillary Thyroid Carcinoma. Thyroid 2020;30:885-97.

46. Vickers AJ, Elkin EB. Decision curve analysis: a novel method for evaluating prediction models. Med Decis Making 2006;26:565-74.

Table S1 Major packages of R software used in this study

\begin{tabular}{lc}
\hline Functions & R package \\
\hline Data Cleansing & fastStat \\
LASSO regression & glmnet \\
Univariate logistic regression analysis & glm \\
ROC and AUC & pROC OptimalCutpoints \\
For ROC analysis to determine optimal cutoff value & rms \\
Plot calibration curves & rmda \\
DCA & PredictABEL \\
NRI and IDI & O \\
\hline
\end{tabular}


Table S2 Univariate Logistic Regression Analysis of MVI Presence in the Training Cohort

\begin{tabular}{|c|c|c|c|}
\hline Variable & $\mathrm{P}$ Value & OR & $(95 \% \mathrm{Cl})$ \\
\hline Age, y & 0.001 & 0.977 & $0.964-0.991$ \\
\hline Gender, male vs. female & 0.076 & 1.445 & $0.962-2.17$ \\
\hline TB, $>20.4$ vs. $\leq 20.4 \mu \mathrm{mol} / \mathrm{L}$ & 0.899 & 0.967 & $0.58-1.615$ \\
\hline $\mathrm{DB},>6.8$ vs. $\leq 6.8 \mu \mathrm{mol} / \mathrm{L}$ & 0.683 & 1.077 & $0.754-1.54$ \\
\hline ALB, $>35-55$ vs. $\leq 35-55 \mathrm{~g} / \mathrm{L}$ & 0.945 & 1.025 & $0.502-2.096$ \\
\hline ALT, $>50$ vs. $\leq 50 \mathrm{U} / \mathrm{L}$ & 0.75 & 1.069 & $0.71-1.61$ \\
\hline AST, $>40$ vs. $\leq 40 \mathrm{U} / \mathrm{L}$ & 0.004 & 1.774 & $1.198-2.627$ \\
\hline ALP $>125$ vs. $\leq 125 \mathrm{U} / \mathrm{L}$ & 0.001 & 4.435 & $2.502-7.861$ \\
\hline GGT $>60$ vs. $\leq 60 \mathrm{U} / \mathrm{L}$ & 0.001 & 1.641 & $1.212-2.222$ \\
\hline $\mathrm{TC},>5.2$ vs. $\leq 5.2 \mathrm{mmol} / \mathrm{L}$ & 0.063 & 1.518 & $0.978-2.355$ \\
\hline $\mathrm{TG},>60$ vs. $\leq 60 \mathrm{U} / \mathrm{L}$ & 0.012 & 1.772 & $1.135-2.767$ \\
\hline ApoA1, >1.7 vs. $\leq 1.7 \mathrm{~g} / \mathrm{L}$ & 0.194 & 0.751 & $0.488-1.157$ \\
\hline ApoB1, >1.55 vs. $\leq 1.55 \mathrm{~g} / \mathrm{L}$ & 0.023 & 4.505 & $1.229-16.511$ \\
\hline ApoE, $>53$ vs. $\leq 53$ mg/L & 0.465 & 1.144 & $0.798-1.639$ \\
\hline $\mathrm{Cr},>115$ vs. $\leq 115 \mu \mathrm{mol} / \mathrm{L}$ & 0.274 & 0.521 & $0.162-1.676$ \\
\hline Glu, $>5.6$ vs. $\leq 5.6 \mathrm{mmol} / \mathrm{L}$ & 0.1 & 1.335 & $0.946-1.883$ \\
\hline \multicolumn{4}{|l|}{ AFP, ng/ml } \\
\hline $20-400$ vs. $\leq 20$ & 0.003 & 1.725 & $1.2-2.481$ \\
\hline$\geq 400$ vs. $20-400$ & 0.001 & 4.257 & $2.886-6.279$ \\
\hline CEA, $>5 v s . \leq 5 \mathrm{ng} / \mathrm{ml}$ & 0.171 & 0.718 & $0.446-1.154$ \\
\hline CA199, >34 vs. $\leq 34 \mathrm{U} / \mathrm{ml}$ & 0.214 & 1.274 & $0.869-1.868$ \\
\hline PLT, $\leq 125$ vs. $>12510^{\wedge} / \mathrm{L}$ & 0.351 & 0.863 & $0.633-1.176$ \\
\hline PT, $>13$ vs. $\leq 13$ seconds & 0.651 & 1.14 & $0.647-2.009$ \\
\hline \multicolumn{4}{|l|}{ PIVKA-II, mAU/ml } \\
\hline $40-400$ vs. $\leq 400$ & 0.001 & 2.179 & $1.537-3.088$ \\
\hline$\geq 400$ vs. $20-400$ & 0.001 & 5.301 & $3.417-8.223$ \\
\hline \multicolumn{4}{|l|}{ HBV DNA load, IU/ml } \\
\hline$>10^{\wedge}$ vs. $\leq 10^{\wedge 4}$ & 0.152 & 1.253 & $0.92-1.705$ \\
\hline HCV, Yes vs. No & 0.845 & 1.318 & $0.082-21.156$ \\
\hline Child-Pugh class, A vs. B & 0.623 & 1.542 & $0.234-2.223$ \\
\hline No. of tumors, Solitary vs. Multiple & 0.027 & 1.632 & $1.057-2.519$ \\
\hline \multicolumn{4}{|l|}{ Pseudo-capsule } \\
\hline III-defined vs. well-defined & 0.004 & 0.645 & $0.476-0.873$ \\
\hline Tumor diameter, cm & 0.001 & 1.856 & $1.54-2.238$ \\
\hline Cirrhosis, Yes vs. No & 0.697 & 0.942 & $0.699-1.27$ \\
\hline \multicolumn{4}{|l|}{ Tumor boundary } \\
\hline non-smooth vs. smooth & 0.247 & 0.827 & $0.6-1.141$ \\
\hline \multicolumn{4}{|l|}{ tumor growth pattern } \\
\hline irregular vs. regular & 0.001 & 2.627 & $1.935-3.566$ \\
\hline \multicolumn{4}{|l|}{ Intratumor inhomogeneous } \\
\hline present vs. absent & 0.001 & 2.501 & $1.718-3.641$ \\
\hline \multicolumn{4}{|l|}{ Arterial enhancement } \\
\hline hypo-/mild vs. hyper- & 0.346 & 2.23 & $0.774-3.42$ \\
\hline Washout, absent vs. present & 0.127 & 1.02 & $0.33-1.26$ \\
\hline
\end{tabular}




\section{A Training cohort}

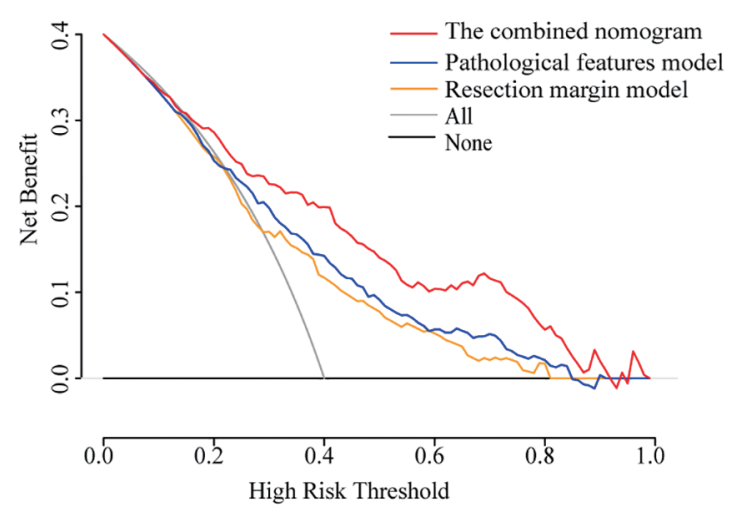

\section{B Validation cohort}

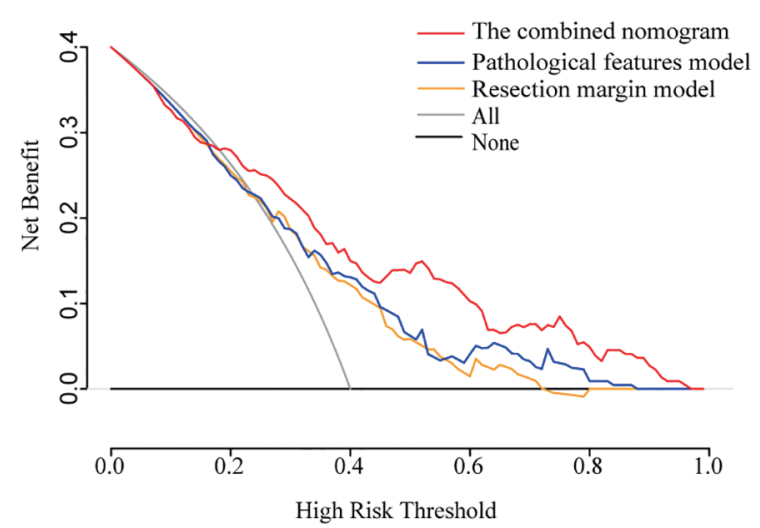

Figure S1 The DCA analysis of the nomogram, pathological features model, resection margin model in recurrence prediction of HCC in the training (A) and validation cohort (B). The DCA shows that present nomogram derived from this study (red curve) predicting recurrence of HCC provides a greater benefit than the pathological features model (green curve) and resection margin model alone (orange curve) in previous studies within 0 to 0.8 threshold probability.

Table S3 Evaluation of the models with respect to NRI and IDI

\begin{tabular}{|c|c|c|c|c|c|c|}
\hline \multirow[b]{2}{*}{ Characteristic } & \multicolumn{3}{|c|}{ Training cohort } & \multicolumn{3}{|c|}{ Validation cohort } \\
\hline & $\begin{array}{l}\text { Categorical } \\
\mathrm{NRI}(95 \% \mathrm{Cl})\end{array}$ & $\begin{array}{l}\text { Continuous } \\
\mathrm{NRI}(95 \% \mathrm{Cl})\end{array}$ & IDI (95\% Cl) & $\begin{array}{l}\text { Categorical } \\
\text { NRI }(95 \% \mathrm{Cl})\end{array}$ & $\begin{array}{l}\text { Continuous } \\
\text { NRI }(95 \% \mathrm{Cl})\end{array}$ & IDI (95\% Cl) \\
\hline \multicolumn{7}{|l|}{ The combined model, vs. } \\
\hline Hematological test model & $0.379(0.284-0.474)$ & $0.7954(0.633-0.958)$ & $0.152(0.119-0.184)$ & $0.28(0.13-0.43)$ & $0.64(0.381-0.898)$ & $0.103(0.06-0.146)$ \\
\hline Resection margin model & $0.337(0.239-0.435)$ & $0.719(0.553-0.884)$ & $0.125(0.096-0.155)$ & $0.367(0.205-0.529)$ & $0.817(0.568-1.067)$ & $0.0 .214(0.157-0.27)$ \\
\hline$P$ value & $\mathrm{P}<0.001^{\star \star \star}$ (both) & $\mathrm{P}<0.001^{\star \star \star}$ (both) & $\mathrm{P}<0.001^{\star \star \star}$ (both) & $\mathrm{P}=0.001^{\star \star \star}$, (both) & $P<0.001^{* \star *}$ (both) & $P<0.001^{\star \star \star}$ (both) \\
\hline
\end{tabular}

